\title{
Antiferromagnetism and single-particle properties in the two-dimensional half-filled Hubbard model: a non-linear sigma model approach
}

\author{
K. Borejsza and N. Dupuis \\ Laboratoire de Physique des Solides, CNRS UMR 8502, \\ Université Paris-Sud, 91405 Orsay, France
}

(Dated: December 4, 2003)

\begin{abstract}
We describe a low-temperature approach to the two-dimensional half-filled Hubbard model which allows us to study both antiferromagnetism and single-particle properties. This approach ignores amplitude fluctuations of the antiferromagnetic (AF) order parameter and is valid below a crossover temperature $T_{X}$ which marks the onset of AF short-range order. Directional fluctuations (spin waves) are described by a non-linear sigma model $(\mathrm{NL} \sigma \mathrm{M})$ that we derive from the Hubbard model. The parameters of the NL $\sigma \mathrm{M}$, the spin stiffness and the spin-wave velocity, are calculated as a function of the Coulomb repulsion $U$. The NL $\sigma \mathrm{M}$ is solved by a saddle-point approximation within the $\mathrm{CP}^{1}$ representation where the Néel field is parametrized by two Schwinger bosons. At zero temperature, there is always Bose condensation of the Schwinger bosons, which signals AF longrange order for any value of the Coulomb repulsion. At finite temperature, the AF long-range order is suppressed (in agreement with the Mermin-Wagner theorem), but the AF correlation length remains exponentially large. In the $\mathrm{CP}^{1}$ representation, the fermion field is naturally expressed as the product of a Schwinger boson and a pseudo-fermion whose spin is quantized along the (fluctuating) Néel field. This allows us to write the fermion Green's function as the product (in direct space) of the Schwinger boson propagator (which is derived from the NL $\sigma \mathrm{M}$ ) and the pseudo-fermion propagator. At zero temperature and weak coupling, our results are typical of a Slater antiferromagnet. The AF gap is exponentially small; there are well-defined Bogoliubov quasi-particles (QP's) (carrying most of the spectral weight) coexisting with a high-energy incoherent excitation background. As $U$ increases, the Slater antiferromagnet progressively becomes a Mott-Heisenberg antiferromagnet. The Bogoliubov bands evolve into Mott-Hubbard bands separated by a large AF gap. A significant fraction of spectral weight is transferred from the Bogoliubov QP's to incoherent excitations. At finite temperature, there is a metal-insulator transition between a pseudogap phase at weak coupling and a MottHubbard insulator at strong coupling. Finally, we point out that our results straightforwardly translate to the half-filled attractive Hubbard model, where the $\mathbf{q}=(\pi, \pi)$ charge and $\mathbf{q}=0$ pairing fluctuations combine to form an order parameter with $\mathrm{SO}(3)$ symmetry.
\end{abstract}

PACS numbers: 71.10.Fd,71.10.Hf,71.27.+a

\section{INTRODUCTION}

The Hubbard mode $\mathrm{e}^{1,2.3 .4}$ and its generalizations play a key role in the description of strongly correlated fermion systems such as high- $T_{c}$ superconductors, heavy fermions systems, or organic conductors $\stackrel{\underline{5}}{\underline{\underline{n}}}$ Despite its simplicity (the model is defined by two parameters, the inter-site hopping amplitude $t$ and the local Coulomb interaction $U$, and the symmetry of the lattice), exact solutions or well-controlled approximations exist only in a few special cases like in one-dimension ${ }^{6}$ (1D) or in the limit of infinite dimension ?

It is now well established that the ground state of the half-filled Hubbard model on a cubic or square lattice has antiferromagnetic (AF) long-range order ${ }^{8.9}$ In the weakcoupling limit $(U \ll 4 t)$, a Fermi surface instability gives rise to a spin-density-wave ground state as first suggested by Slater ${ }^{10}$ The AF long-range order produces a gap in the quasi-particle (QP) excitation spectrum so that the system becomes insulating below the AF transition temperature. In the strong-coupling regime $(U \gg 4 t)$, fermions are localized by the strong Coulomb repulsion (Mott-Hubbard localization), thus creating local (magnetic) moments on the lattice sites that are well described by the Heisenberg model ${ }^{4.11}$ These local moments order at low temperature and give rise to a Mott-Heisenberg antiferromagnet.

The main difference between Slater and MottHeisenberg antiferromagnets lies in the existence or absence of preformed local (magnetic) moments above the Néel temperature $T_{\mathrm{N}}{ }^{4}$ In the weak-coupling limit, we expect a Fermi liquid phase down to temperatures very close to $T_{\mathrm{N}}$ where critical AF fluctuations start to grow. In the strong-coupling limit, the system is insulating both above (Mott-Hubbard insulator) and below (MottHeisenberg antiferromagnet) the Néel temperature.

This simple view, while correct in 3D, breaks down in 2D. In 2D systems, thermal (classical) fluctuations preclude a finite-temperature AF phase transition, and the phase transition occurs at $T_{\mathrm{N}}=0$ in agreement with the Mermin-Wagner theorem ${ }^{12}$ Nevertheless, below a crossover temperature $T_{X}$, the system enters a renormalized classical regime where AF fluctuations start to grow exponentially. Below $T_{X}$, the Fermi-liquid description breaks down even at weak coupling, although the system remains metallic. Instead of well-defined Landau's QP's, the fermion spectral function $\mathcal{A}(\mathbf{k}, \omega)$ exhibits two (broadened) peaks separated by a pseudogap. 
The existence of a pseudogap at weak coupling is best understood by considering the zero-temperature limit. At zero temperature, $\mathcal{A}(\mathbf{k}, \omega)$ is expected to exhibit two peaks corresponding to the Bogoliubov QP's as in the Hartree-Fock (HF) theory. These two peaks are separated by the $\mathrm{AF}$ gap which is due to the presence of magnetic long-range order. At any finite temperature, the AF long-range order disappears in 2D. However, by continuity, the two-peak structure in $\mathcal{A}(\mathbf{k}, \omega)$ cannot disappear as soon as we raise the temperature. As pointed out in Ref. 13, the only possible scenario is that at finite by low temperature, the fermion spectral function exhibits two broadened peaks, which are precursors of the zero-temperature Bogoliubov QP's, separated by a pseudogap. At strong coupling, the zero-temperature gap survives at finite temperature since the system is a MottHubbard insulator.

The simplest description of the AF ground state of the 2D half-filled Hubbard model is based on the HF theory. It is known that the HF theory remains meaningful even at large $U$. In particular, spin-wave modes obtained from the Heisenberg model with an exchange coupling $J=4 t^{2} / U$ can be reproduced from a randomphase-approximation (RPA) calculation about the AF HF solution.14.15.16.17 The influence of the spin-wave modes on the fermionic excitations has been studied within one-loop ${ }^{18}$ and self-consistent one-loop ${ }^{19.20}$ approximations. A QP picture for the coherent motion of a particle or a hole appears to be still valid. However, AF quantum fluctuations lead to a significant reduction of the Bogoliubov QP spectral weight, with a concomitant redistribution of spectral intensity into incoherent excitations, and a strong renormalization of the AF gap. These conclusions are supported by numerical work on the Hubbard model ${ }^{21.22 .23}$ and, in the strong-coupling limit, by analytical or numerical analysis of the $t$ - $J$ model ${ }^{24}$

In spite of its success at zero temperature, the HF theory fails in $2 \mathrm{D}$ since it predicts AF long-range order at finite temperature. In the weak-coupling limit, alternative approaches, which do satisfy the Mermin-Wagner theorem, have been proposed: Moriya's self-consistentrenormalized theory ${ }^{14.25 .26}$ the fluctuation exchange approximation (FLEX) ${ }^{27}$ or the two-particle self-consistent theory. ${ }^{13}$ None of these approaches gives a unified description of the magnetic properties of the 2D Hubbard model at finite temperature, both at weak and strong coupling. At strong coupling, in the Mott-Hubbard insulating state, spin degrees of freedom are usually described by the Heisenberg model for which various methods are available $11,28.29$

Beside their limitation to the weak-coupling regime, these approaches are also unable to account for the strong suppression of the amplitude fluctuations of the AF order parameter at low temperature and therefore essentially describe Gaussian spin fluctuations. Below the crossover temperature $T_{X}$, amplitude fluctuations are indeed frozen and only directional fluctuations [i.e. (transverse) spin waves] survive at low en- ergy. The calculation of the single-particle Green's function usually relies on a paramagnon-like self-energy describing free fermions that couple to Gaussian order parameter fluctuations. ${ }^{13.27 .30 .31 .32}$ This kind of approach has been originally introduced by Lee, Rice and Anderson to explain the suppression of the density of states associated with order parameter fluctuations near a charge-density-wave instability. ${ }^{33}$ It has been since studied by many authors, in one and

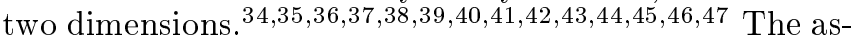
sumption of Gaussian spin fluctuations leads to an overestimation of the fermion density of states at low energy. 48 Moreover, the artificial presence of amplitude fluctuations does not allow to reach the correct $T \rightarrow 0$ limit. ${ }^{36.49}$ The effect of "directional" (i.e. phase) fluctuations of a complex order parameter on the fermion density of states has been studied both for incommensurate 1D Peierls systems 50.51 and $2 \mathrm{D}$ superconductors ${ }^{52}$ Transverse spinwave fluctuations in the finite-temperature 2D Hubbard model have not received as much attention so far.

On the experimental side, antiferromagnetism and pseudogaps are ubiquitous in low-dimensional strongly correlated fermion systems. Pseudogaps were first observed in quasi-1D systems near a charge-densitywave instability. ${ }^{33.53}$ More recently, a pseudogap has been observed in the metallic phase of high- $T_{c}$ superconductors 54.55 Whether the pseudogap in these systems is of magnetic or pairing origin is still a matter of intense debate.

In this paper, we describe a theoretical approach which provides a unified view of the $2 \mathrm{D}$ half-filled Hubbard model at low temperature (including $T=0$ ) and for any value of the Coulomb repulsion ${ }^{56}$ It is based on a non-linear sigma model (NL $\sigma \mathrm{M})$ description of spin fluctuations. At zero temperature, our theory describes the evolution from a Slater $(U \ll 4 t)$ to a Mott-Heisenberg $(U \gg 4 t)$ antiferromagnet. At finite temperature, it predicts a pseudogap at weak-coupling due to strong AF fluctuations, and a Mott-Hubbard gap at strong coupling. Since it takes into account only directional fluctuations of the AF order parameter, it is valid for $T \ll T_{X}$, where $T_{X}$ is a crossover temperature which marks the onset of $\mathrm{AF}$ short-range order. In Ref. 49, one of the present authors reported a calculation of the fermion spectral function in the weak-coupling limit of the Hubbard model using a NL $\sigma \mathrm{M}$ description of spin fluctuations. However, the limitations encountered by previous approaches could not be overcome.

As first shown by Schulz, $\frac{57}{5}$ spin fluctuations in the 2D Hubbard model at low temperature can be described by a NL $\sigma \mathrm{M}$ for any value of the Coulomb repulsion. 58 In Sec. II we give a detailed derivation of the NL $\sigma \mathrm{M}$ starting from the Hubbard model. The parameters of the NL $\sigma \mathrm{M}$, the bare spin stiffness $\rho_{s}^{0}$ and the spin-wave velocity $c$, are calculated as a function of the ratio $U / t$. For $U \gg 4 t$, we recover the $\mathrm{NL} \sigma \mathrm{M}$ derived from the Heisenberg model with an exchange coupling $J=4 t^{2} / U$. In Sec. III we introduce the $\mathrm{CP}^{1}$ representation of the 
$\mathrm{NL} \sigma \mathrm{M}$ where the Néel field (giving the direction of the local AF order) is expressed in terms of two Schwinger bosons. This allows a simple saddle-point solution 11 from which we obtain the magnetic phase diagram of the $2 \mathrm{D}$ Hubbard model. At zero temperature, there is condensation of the Schwinger bosons for any value of $U$, which signals the presence of AF long-range order. At finite temperature, the system is disordered by thermal fluctuations, but the $\mathrm{AF}$ correlation length remains exponentially large below a crossover temperature $T_{X}$ (renormalized classical regime ${ }^{28}$ ). In Sec. IV we study the fermion spectral properties. The fermion is written as the product of a Schwinger boson and a pseudo-fermion whose spin is quantized along the (fluctuating) Néel field. Such a decomposition is reminiscent of slave-boson ${ }^{59}$ or slavefermion ${ }^{60.61 .62}$ theories ${ }^{63}$ It allows us to approximate the fermion Green's function by the product (in direct space) of the Schwinger boson propagator (which is obtained from the $\mathrm{NL} \sigma \mathrm{M})$ and the HF fermionic propagator. At weak-coupling ( $U \ll 4 t)$ and zero temperature, our results clearly describe a Slater antiferromagnet. The AF gap $2 \Delta_{0} \sim t e^{-2 \pi \sqrt{t / U}}$ is exponentially small. As in the HF theory, there are well-defined Bogoliubov QP's. However, because of AF quantum fluctuations, their spectral weight is reduced by a factor $n_{0}\left(0<n_{0}<1\right)$ which is given by the fraction of condensed Schwinger bosons in the ground state. The missing weight $\left(1-n_{0}\right)$ is transferred to incoherent excitations at higher energy $\left(1-n_{0} \ll 1\right.$ when $\left.U \ll 4 t\right)$. As $U$ increases, the AF gap increases and spectral weight is progressively transferred from the Bogoliubov QP's to the incoherent excitation background. At strong coupling $(U \gg 4 t)$, our results are typical of a Mott-Heisenberg antiferromagnet. The AF gap $2 \Delta_{0}$ is of order $U$. The incoherent excitation background carries a significant fraction of spectral weight (i.e. $n_{0}$ and $1-n_{0}$ are of the same order) and extends over an energy scale of order $J=4 t^{2} / U$ above the Bogoliubov QP energy $\pm E_{\mathbf{k}}$. At finite temperature, the Bogoliubov QP's disappear $\left(n_{0}=0\right.$ in the absence of Bose condensation) and only incoherent excitations survive. Nevertheless, precursors of the zero-temperature Bogoliubov QP's show up as sharp peaks at $\pm E_{\mathbf{k}}$ in the spectral function $\mathcal{A}(\mathbf{k}, \omega)$, with a width of order $T$. We show that these peaks continuously evolve into the zerotemperature Bogoliubov QP peaks as $T \rightarrow 0$. This ensures that the spectral function $\mathcal{A}(\mathbf{k}, \omega)$ is continuous at the $T_{\mathrm{N}}=0$ phase transition. The high-energy incoherent excitation background is little affected by a finite temperature, but the presence of thermal AF fluctuations gives rise to fermionic states below the zero-temperature $\mathrm{AF}$ gap $\Delta_{0}$. At weak coupling, the gap is completely filled and replaced by a pseudogap. At strong coupling, the zero-temperature gap survives at finite temperature and the system is a Mott-Hubbard insulator.

On the basis of a numerical calculation in the framework of the dynamical cluster approximation, Moukouri and Jarrell have called into question the existence of a Slater scenario in the 2D half-filled Hubbard model ${ }^{64.65 .66}$ They argue that the system is always a Mott-Hubbard insulator at low (but finite) temperature even at weak coupling. We will show that their results are not in contradiction with a Slater scenario at weak coupling, but merely reflect the strong suppression of the density of states due to the pseudogap (Sec. IVC).

At half-filling, the repulsive Hubbard model can be mapped exactly onto the attractive model by a canonical transformation ${ }^{67}$ This transformation maps the $\mathbf{q}=$ $(\pi, \pi)$ spin correlations of the repulsive model onto the $\mathbf{q}=0$ pairing and $\mathbf{q}=(\pi, \pi)$ charge correlations of the attractive model, but leaves the single-particle Green's function and the spectral function $\mathcal{A}(\mathbf{k}, \omega)$ invariant. Thus the results obtained in this paper apply also to the attractive Hubbard model, but with a different physical meaning (Sec. V). At zero temperature, there is superconducting and charge-density-wave long-range orders. As the attractive interaction strength increases, there is a smooth crossover from a BCS to a Bose-Einstein behavior. At finite temperature, the weak-coupling pseudogap is due to strong pairing and charge fluctuations, whereas the strong-coupling gap is a consequence of the presence of preformed particle-particle pairs.

\section{DERIVATION OF THE NL $\sigma \mathrm{M}$}

The Hubbard model is defined by the Hamiltonian

$$
H=-\sum_{\mathbf{r}, \sigma} c_{\mathbf{r} \sigma}^{\dagger}(\hat{t}+\mu) c_{\mathbf{r} \sigma}+U \sum_{\mathbf{r}} c_{\mathbf{r} \uparrow}^{\dagger} c_{\mathbf{r} \uparrow} c_{\mathbf{r} \downarrow}^{\dagger} c_{\mathbf{r} \downarrow}
$$

where $\hat{t}$ is the nearest-neighbor hopping operator:

$$
\hat{t} c_{\mathbf{r} \sigma}=t\left(c_{\mathbf{r}+\mathbf{e}_{x} \sigma}+c_{\mathbf{r}-\mathbf{e}_{x} \sigma}+c_{\mathbf{r}+\mathbf{e}_{y} \sigma}+c_{\mathbf{r}-\mathbf{e}_{y} \sigma}\right) .
$$

At half-filling the chemical potential $\mu$ equals $U / 2$. $e_{x}$ and $e_{y}$ denote unit vectors along the $x$ and $y$ directions. $c_{\mathbf{r} \sigma}^{\dagger}\left(c_{\mathbf{r} \sigma}\right)$ creates (annihilates) a fermion of spin $\sigma$ at the lattice site $\mathbf{r}$. We take the lattice spacing equal to unity and set $\hbar=k_{\mathrm{B}}=1$ throughout the paper.

We can represent the partition function of the system as a path integral over Grassmann fields $\psi_{\mathbf{r} \sigma}, \psi_{\mathbf{r} \sigma}^{\star}$. The action can be written as $S_{\text {kin }}+S_{\text {int }}$ with

$$
\begin{aligned}
S_{\mathrm{kin}} & =\int_{0}^{\beta} d \tau \sum_{\mathbf{r}} \Psi_{\mathbf{r}}^{\dagger}\left(\partial_{\tau}-\mu-\hat{t}\right) \Psi_{\mathbf{r}}, \\
S_{\mathrm{int}} & =U \int_{0}^{\beta} d \tau \sum_{\mathbf{r}} \psi_{\mathbf{r} \uparrow}^{\star} \psi_{\mathbf{r} \uparrow} \psi_{\mathbf{r} \downarrow}^{\star} \psi_{\mathbf{r} \downarrow},
\end{aligned}
$$

where $\beta=1 / T$ is the inverse temperature. In the kinetic action $S_{\text {kin }}$ we have used the spinor representation $\Psi=\left(\psi_{\uparrow}, \psi_{\downarrow}\right)^{T}$. To describe collective spin and charge fluctuations, we introduce auxiliary fields. The standard approach is to write the interaction part of the action as $\psi_{\mathbf{r} \uparrow}^{\star} \psi_{\mathbf{r} \uparrow} \psi_{\mathbf{r} \downarrow}^{\star} \psi_{\mathbf{r} \downarrow}=\frac{1}{4}\left(\Psi_{\mathbf{r}}^{\dagger} \Psi_{\mathbf{r}}\right)^{2}-\frac{1}{4}\left(\Psi_{\mathbf{r}}^{\dagger} \sigma_{3} \Psi_{\mathbf{r}}\right)^{2}$, and to perform a Hubbard-Stratonovich transformation 
by means of two real auxiliary fields $\Delta_{c \mathbf{r}}$ and $\Delta_{s \mathbf{r}}$. Although this procedure recovers the standard mean-field (or HF) theory of the Neel state within a saddle-point approximation, it leads to a loss of spin rotation invariance and does not allow to obtain the spin-wave Goldstone modes. Fluctuations of $\Delta_{c \mathbf{r}}$ and $\Delta_{s \mathbf{r}}$ correspond to gapped amplitude modes. Alternatively, one could write $S_{\text {int }}$ in an explicitly spin-rotation invariant form, e.g. $\quad \psi_{\mathbf{r} \uparrow}^{\star} \psi_{\mathbf{r} \uparrow} \psi_{\mathbf{r} \downarrow}^{\star} \psi_{\mathbf{r} \downarrow}=-\frac{1}{6}\left(\Psi_{\mathbf{r}}^{\dagger} \boldsymbol{\sigma} \Psi_{\mathbf{r}}\right)^{2}\left(\boldsymbol{\sigma}=\left(\sigma_{1}, \sigma_{2}, \sigma_{3}\right)\right.$ denotes the Pauli matrices), and use a vector HubbardStratonovich field. Such decompositions, however, do not reproduce the $\mathrm{HF}$ results at the saddle-point level. ${ }^{57} \mathrm{As}$ noted earlier,$\stackrel{57.68}{~ t h i s ~ d i f f i c u l t y ~ c a n ~ b e ~ c i r c u m v e n t e d ~ b y ~}$ using the decomposition

$$
\psi_{\mathbf{r} \uparrow}^{\star} \psi_{\mathbf{r} \uparrow} \psi_{\mathbf{r} \downarrow}^{\star} \psi_{\mathbf{r} \downarrow}=\frac{1}{4}\left(\Psi_{\mathbf{r}}^{\dagger} \Psi_{\mathbf{r}}\right)^{2}-\frac{1}{4}\left(\Psi_{\mathbf{r}}^{\dagger} \boldsymbol{\sigma} \cdot \Omega_{\mathbf{r}} \Psi_{\mathbf{r}}\right)^{2},
$$

where $\boldsymbol{\Omega}_{\mathbf{r}}$ is a site- and time-dependent unitary vector. Spin-rotation invariance is made explicit by performing an angular integration over $\boldsymbol{\Omega}_{\mathbf{r}}$ at each site and time (with a measure normalized to unity). The HubbardStratonovich transformation then reads

$$
\begin{aligned}
e^{-S_{\mathrm{int}}}= & \int \mathcal{D}\left[\Delta_{c}, \Delta_{s}, \boldsymbol{\Omega}\right] \\
& \times e^{-\int_{0}^{\beta} d \tau \sum_{\mathbf{r}}\left[\frac{1}{U}\left(\Delta_{c \mathbf{r}}^{2}+\Delta_{s \mathbf{r}}^{2}\right)-\Psi_{\mathbf{r}}^{\dagger}\left(i \Delta_{c \mathbf{r}}+\Delta_{s \mathbf{r}} \boldsymbol{\sigma} \cdot \boldsymbol{\Omega}_{\mathbf{r}}\right) \Psi\right.}(6 .)
\end{aligned}
$$

Eq. (6) corresponds to an "amplitude-direction" representation, where the magnetic order parameter field is given by $\Delta_{s \mathbf{r}} \Omega_{\mathbf{r}}$. The HF theory is now recovered from a saddle-point approximation over the auxiliary fields $\Delta_{c \mathbf{r}}$, $\Delta_{s \mathbf{r}}$ and $\boldsymbol{\Omega}_{\mathbf{r}}$ (Sec. IIA). Spin-wave excitations can then be obtained by considering small fluctuations of the $\boldsymbol{\Omega}_{\mathbf{r}}$ field about its saddle-point value. In Sec. III we show that the amplitude-direction representation (6) allows to go beyond the Néel-ordered HF state and derive an effective action for the $\boldsymbol{\Omega}_{\mathbf{r}}$ field.

\section{A. HF theory}

Making the ansatz of an antiferromagnetic order, i.e. taking constant auxiliary fields $\Delta_{c \mathbf{r}}=\Delta_{c}, \Delta_{s \mathbf{r}}=\Delta$ and a staggering vector $\Omega_{\mathbf{r}}=(-1)^{\mathbf{r}} \mathbf{u}_{z}$ parallel to the $z$ axis, one obtains the saddle-point equations

$$
\begin{aligned}
i \Delta_{c} & =-\frac{U}{2}\left\langle\Psi_{\mathbf{r}}^{\dagger} \Psi_{\mathbf{r}}\right\rangle \\
\Delta & =\frac{U}{2}(-1)^{\mathbf{r}}\left\langle\Psi_{\mathbf{r}}^{\dagger} \sigma_{3} \Psi_{\mathbf{r}}\right\rangle .
\end{aligned}
$$

At half-filling, the saddle-point value $i \Delta_{c}=-U / 2$ cancels the chemical potential term in (3). The HF action is quadratic,

$$
S_{\mathrm{HF}}=\int_{0}^{\beta} d \tau \sum_{\mathbf{r}} \Psi_{\mathbf{r}}^{\dagger}\left(\partial_{\tau}-\hat{t}-(-1)^{\mathbf{r}} \Delta \sigma_{3}\right) \Psi_{\mathbf{r}},
$$

and can easily be diagonalized. Due to the translational symmetry breaking there is unit cell doubling. In the reduced Brillouin zone scheme $\left(\left|k_{x}\right|+\left|k_{y}\right| \leq \pi\right)$ elementary excitations are exhausted by two bands of Bogoliubov QP's at energies $\pm E_{\mathbf{k}}= \pm \sqrt{\Delta^{2}+\epsilon_{\mathbf{k}}^{2}}, \epsilon_{\mathbf{k}}=$ $-2 t\left(\cos k_{x}+\cos k_{y}\right)$ being the energy of free fermions.

Using the HF action (9) one obtains the HF singleparticle Green's function

$$
\begin{aligned}
-\left\langle\phi_{\mathbf{k} \omega \sigma} \phi_{\mathbf{k}^{\prime} \omega^{\prime} \sigma^{\prime}}^{\star}\right\rangle & =\delta_{\omega, \omega^{\prime}} \delta_{\sigma, \sigma^{\prime}} \mathcal{G}_{\sigma}^{\mathrm{HF}}\left(\mathbf{k}, \mathbf{k}^{\prime}, \omega\right), \\
\mathcal{G}_{\sigma}^{\mathrm{HF}}\left(\mathbf{k}, \mathbf{k}^{\prime}, \omega\right) & =-\delta_{\mathbf{k}, \mathbf{k}^{\prime}} \frac{i \omega+\epsilon_{\mathbf{k}}}{\omega^{2}+E_{\mathbf{k}}^{2}}+\delta_{\mathbf{k}, \mathbf{k}^{\prime}+\pi} \frac{\sigma \Delta}{\omega^{2}+E_{\mathbf{k}}^{2}},
\end{aligned}
$$

where $\pi=(\pi, \pi)$ and $\omega \equiv(2 n+1) \pi T$ ( $n$ integer) is a fermionic Matsubara frequency. The propagator (10) makes it in turn possible to give an explicit form to the gap equation (8):

$$
\frac{1}{U}=\int_{\mathbf{k}} \frac{\tanh \left(\frac{\beta E_{\mathbf{k}}}{2}\right)}{2 E_{\mathbf{k}}} .
$$

We use the notation $\int_{\mathbf{k}}=\int_{-\pi}^{\pi} \int_{-\pi}^{\pi} \frac{d k_{x}}{2 \pi} \frac{d k_{y}}{2 \pi}$. Eq. (12) predicts a phase transition at a finite temperature $T_{\mathrm{N}}^{\mathrm{HF}}$, which is exponentially small at weak coupling and approaches $U / 4$ at strong coupling. Similarly to the transition temperature, the zero-temperature gap $\Delta_{0}$ tends to $U / 2$ at strong coupling and is exponentially small at weak coupling:

$$
\Delta_{0} \simeq 32 t e^{-2 \pi \sqrt{t / U}}
$$

\section{B. Spin fluctuations}

In $2 \mathrm{D}$, the $\mathrm{HF}$ theory breaks down at finite temperature, since it predicts AF long-range order below $T_{\mathrm{N}}^{\mathrm{HF}}$. Nevertheless, the HF transition temperature bears a physical meaning as a crossover temperature below which the amplitude of the AF order parameter takes a well-defined value. This is sometimes interpreted as the appearance of local moments with an amplitude $\Delta_{0} / U$. Note that at weak coupling the "local" moments can be defined only at length-scale of order $\xi_{0} \sim t / \Delta_{0}$, which corresponds to the size of bound particle-hole pairs in the HF ground state. Thus, stricto sensu, local moments form only in the strong-coupling limit when $\xi_{0} \sim 1$.

Below $T_{\mathrm{N}}^{\mathrm{HF}}$, it is natural to neglect amplitude fluctuations of the AF order parameter and derive an effective action of the $\boldsymbol{\Omega}_{\mathbf{r}}$ field by integrating out the fermionic degrees of freedom. We call $T_{\mathrm{X}}$ the crossover temperature below which AF short-range order appears. As will be shown subsequently, in the weak coupling limit $T_{\mathrm{X}} \sim T_{\mathrm{N}}^{\mathrm{HF}}$, whereas at strong coupling $T_{\mathrm{X}} \sim J=$ $4 t^{2} / U \ll T_{\mathrm{N}}^{\mathrm{HF}}$. For $T \ll T_{X}$, the amplitude of the AF order parameter can be approximated by its zerotemperature $\mathrm{HF}$ value $\Delta_{0}$. Following Haldane $\stackrel{11.69}{ }$ in 
the presence of $\mathrm{AF}$ short-range order $\left(T \lesssim T_{\mathrm{X}}\right)$ we write

$$
\boldsymbol{\Omega}_{\mathbf{r}}=(-1)^{\mathbf{r}} \mathbf{n}_{\mathbf{r}} \sqrt{1-\mathbf{L}_{\mathbf{r}}^{2}}+\mathbf{L}_{\mathbf{r}} .
$$

$\mathbf{n}$ is a unitary vector representing the Néel field, whereas $\mathbf{L}$ is the canting vector, orthogonal to $\mathbf{n}$, taking account of local ferromagnetic fluctuations. $\mathbf{n}$ is assumed to be slowly-varying and $\mathbf{L}$ to be small. We perform at each site and time a rotation in spin space and introduce a new fermionic field $\Phi_{\mathrm{r}}$ defined by $\Psi_{\mathrm{r}}=R_{\mathrm{r}} \Phi_{\mathrm{r}} . R_{\mathrm{r}}$ is a time- and site-dependent $\mathrm{SU}(2) / \mathrm{U}(1)$ matrix satisfying

$$
\boldsymbol{\sigma} \cdot \mathbf{n}_{\mathbf{r}}=R_{\mathbf{r}} \sigma_{3} R_{\mathbf{r}}^{\dagger}
$$

The above definition means that $\mathcal{R}_{\mathbf{r}}$, the $\mathrm{SO}(3)$ element associated to $R_{\mathbf{r}}$, maps $\mathbf{u}_{z}$ onto $\mathbf{n}_{\mathbf{r}}$. The $\mathrm{U}(1)$ gauge freedom is due to rotations around the $z$ axis, which do not change the physical state of the system. The spin of the pseudo-fermions $\Phi_{\mathbf{r}}$ is quantized along the $\mathbf{n}_{\mathbf{r}}$ axis. In order to express the action in terms of the new spinor variable, it is convenient to make use of the $\mathrm{SU}(2)$ gauge field $A_{\mu \mathbf{r}}=\sum_{\nu=1,2,3} A_{\mu \mathbf{r}}^{\nu} \sigma_{\nu}$ defined as

$$
\begin{aligned}
& A_{0 \mathbf{r}}=-R_{\mathbf{r}}^{\dagger} \partial_{\tau} R_{\mathbf{r}}, \\
& A_{\mu \mathbf{r}}=i R_{\mathbf{r}}^{\dagger} \partial_{\mu} R_{\mathbf{r}}, \quad \mu=x, y .
\end{aligned}
$$

We also define the rotated canting field $\mathbf{l}_{\mathbf{r}}=\mathcal{R}_{\mathbf{r}}^{-1} \mathbf{L}_{\mathbf{r}}$. Given that $\mathcal{R}_{\mathbf{r}}^{-1} \mathbf{n}_{\mathbf{r}}=\mathbf{u}_{z}$ and $\mathbf{L}_{\mathbf{r}} \perp \mathbf{n}_{\mathbf{r}}$, the $\mathbf{l}_{\mathbf{r}}$ vector lies in the $x-y$ plane. Using the identity

$$
\Phi_{\mathbf{r}}^{\dagger} R_{\mathbf{r}}^{\dagger} R_{\mathbf{r}+\mathbf{e}_{\mu}} \Phi_{\mathbf{r}+\mathbf{e}_{\mu}}=\Phi_{\mathbf{r}}^{\dagger} e^{\partial_{\mu}-i A_{\mu \mathbf{r}}} \Phi_{\mathbf{r}}
$$

we re-express the kinetic and interaction parts of the action as

$$
\begin{aligned}
S_{\text {kin }}= & \int_{0}^{\beta} d \tau \sum_{\mathbf{r}} \Phi_{\mathbf{r}}^{\dagger}\left[\partial_{\tau}-A_{0 \mathbf{r}}\right. \\
& \left.-2 t \sum_{\mu=x, y} \cos \left(-i \partial_{\mu}-A_{\mu \mathbf{r}}\right)\right] \Phi_{\mathbf{r}} \\
S_{\text {int }}= & -\Delta_{0} \int_{0}^{\beta} d \tau \sum_{\mathbf{r}} \Phi_{\mathbf{r}}^{\dagger}\left[(-1)^{\mathbf{r}} \sigma_{3} \sqrt{1-\mathbf{l}_{\mathbf{r}}^{2}}\right. \\
& \left.+\mathbf{l}_{\mathbf{r}} \cdot \boldsymbol{\sigma}\right] \Phi_{\mathbf{r}} .
\end{aligned}
$$

In the above expressions, both $\mathbf{l}$ and $A_{\mu}$ are small, since the gauge field is of the order of $\partial_{\mu} \mathbf{n}$. We expand Eqs. (1920) to second order in these variables. To zeroth order, we recover the HF action $S_{\mathrm{HF}}[\Phi]$ defined in (9). The first- and second-order corrections in $A_{\mu}^{\nu}$ yield paramagnetic and diamagnetic terms $S_{\mathrm{p}}$ and $S_{\mathrm{d}}$, respectively. The corrections in $\mathbf{l}$ give first- and second-order ferromagnetic fluctuations $S_{l}$ and $S_{l^{2}}{ }^{70}$

$$
\begin{aligned}
S_{\mathrm{p}} & =-\int_{0}^{\beta} d \tau \sum_{\substack{\mu=0, x, y \\
\nu=1,2,3 \\
\mathbf{r}}} j_{\mu \mathbf{r}}^{\nu} A_{\mu \mathbf{r}}^{\nu} \\
S_{\mathrm{d}} & =\frac{t}{2} \int_{0}^{\beta} d \tau \sum_{\substack{\mu=x, y \\
\nu=1,2,3 \\
\mathbf{r}, 3}} A_{\mu \mathbf{r}}^{\nu} \Phi_{\mathbf{r}}^{\dagger} \cos \left(-i \partial_{\mu}\right) \Phi_{\mathbf{r}}+\text { c.c. }(22)
\end{aligned}
$$

$$
\begin{aligned}
S_{l} & =-\Delta_{0} \int_{0}^{\beta} d \tau \sum_{\substack{\nu=1,2 \\
\mathbf{r}}} l_{\mathbf{r}}^{\nu} j_{0 \mathbf{r}}^{\nu}, \\
S_{l^{2}} & =\frac{\Delta_{0}}{2} \int_{0}^{\beta} d \tau \sum_{\mathbf{r}}(-1)^{\mathbf{r}} \mathbf{l}_{\mathbf{r}}^{2} j_{0 \mathbf{r}}^{3} .
\end{aligned}
$$

The spin-density currents $j_{\mu}^{\nu}$ are defined by

$$
\begin{aligned}
& j_{0 \mathbf{r}}^{\nu}=\Phi_{\mathbf{r}}^{\dagger} \sigma_{\nu} \Phi_{\mathbf{r}} \\
& j_{\mu \mathbf{r}}^{\nu}=t \Phi_{\mathbf{r}}^{\dagger} \sin \left(-i \partial_{\mu}\right) \sigma_{\nu} \Phi_{\mathbf{r}}+\text { c.c. }, \quad \mu=x, y
\end{aligned}
$$

We now derive an effective action for the spin variables $\mathbf{n}$ and $\mathbf{L}$ by integrating out the fermions. Keeping terms up to second order in $A_{\mu}^{\nu}$ and $\mathbf{l}$, the effective action is given by first- and second-order cumulants of the four perturbative terms $S_{\mathrm{p}}, S_{\mathrm{d}}, S_{l}, S_{l^{2}}$ with respect to the HF action:

$$
\begin{aligned}
S_{\mathrm{eff}}[\mathbf{n}, \mathbf{L}]= & \left\langle S_{\mathrm{p}}\right\rangle+\left\langle S_{\mathrm{d}}\right\rangle+\left\langle S_{l}\right\rangle+\left\langle S_{l^{2}}\right\rangle \\
& -\frac{1}{2}\left\langle S_{\mathrm{p}}^{2}\right\rangle_{c}-\frac{1}{2}\left\langle S_{l}^{2}\right\rangle_{c}-\left\langle S_{\mathrm{p}} S_{l}\right\rangle_{c} .
\end{aligned}
$$

Evaluation of the first-order cumulants is straightforward. Defining $\epsilon_{c}$ as the absolute value of the (negative) kinetic energy per site in the HF ground state we have

$$
\begin{aligned}
\left\langle S_{\mathrm{p}}\right\rangle & =-\frac{2 \Delta_{0}}{U} \int_{0}^{\beta} d \tau \sum_{\mathbf{r}}(-1)^{\mathbf{r}} A_{0 \mathbf{r}}^{3}, \\
\left\langle S_{\mathrm{d}}\right\rangle & =\frac{\epsilon_{c}}{4} \int_{0}^{\beta} d \tau \sum_{\substack{\mu=x, y \\
\nu=1,2,3}} A_{\mu \mathbf{r}}^{\nu}{ }^{2}, \\
\left\langle S_{l}\right\rangle & =0 \\
\left\langle S_{l^{2}}\right\rangle & =\frac{\Delta_{0}^{2}}{U} \int_{0}^{\beta} d \tau \sum_{\mathbf{r}} \mathbf{l}_{\mathbf{r}}^{2} .
\end{aligned}
$$

We recognize in equation (28) the usual Berry phase term. Since it is believed to play no role in a twodimensional antiferromagnet $\frac{11}{\underline{11}}$ we will ignore it in the following.

The calculation of the second-order cumulants seems cumbersome at first sight, since it involves (after moving to the Fourier space) the current-current correlation function $\Pi_{\mu \mu^{\prime}}^{\nu \nu^{\prime}}\left(\mathbf{q}, \omega_{\nu} ; \mathbf{q}^{\prime}, \omega_{\nu}^{\prime}\right)=\left\langle j_{\mu}^{\nu}\left(\mathbf{q}, \omega_{\nu}\right) j_{\mu^{\prime}}^{\nu^{\prime}}\left(\mathbf{q}^{\prime}, \omega_{\nu}^{\prime}\right)\right\rangle_{\mathrm{HF}}$. $\left[\omega_{\nu}=\nu 2 \pi T\right.$ ( $\nu$ integer $)$ is a bosonic Matsubara frequency.] In fact, as the correlator stands in front of second-order quantities, we are interested only in its zerofrequency, zero-momentum value $\Pi_{\mu \mu^{\prime}}^{\nu \nu^{\prime}}(\mathbf{0}, 0 ; \mathbf{0}, 0)$ which we denote by $\Pi_{\mu \mu^{\prime}}^{\nu \nu^{\prime}}$. With the exception of $\Pi_{00}^{11}=\Pi_{00}^{22}$ and $\Pi_{x x}^{33}=\Pi_{y y}^{33}$ all these quantities vanish (see Appendix [], so that we obtain

$$
\begin{aligned}
\left\langle S_{\mathrm{p}}^{2}\right\rangle_{c}= & \Pi_{00}^{11} \int_{0}^{\beta} d \tau \sum_{\substack{\mathbf{r} \\
\nu=1,2}} A_{0 \mathbf{r}}^{\nu{ }^{2}} \\
& +\Pi_{x x}^{33} \int_{0}^{\beta} d \tau \sum_{\substack{\mathbf{r} \\
\mu=x, y}} A_{\mu \mathbf{r}}^{3{ }^{2}},
\end{aligned}
$$




$$
\begin{aligned}
\left\langle S_{l}^{2}\right\rangle_{c} & =\Delta_{0}^{2} \Pi_{00}^{11} \int_{0}^{\beta} d \tau \sum_{\substack{\mathbf{r} \\
\nu=1,2}} l_{\mathbf{r}}^{22}, \\
\left\langle S_{\mathrm{p}} S_{l}\right\rangle_{c} & =\Delta_{0} \Pi_{00}^{11} \int_{0}^{\beta} d \tau \sum_{\substack{\mathbf{r} \\
\nu=1,2}} A_{0 \mathbf{r}}^{\nu} l_{\mathbf{r}}^{\nu} .
\end{aligned}
$$

Using the invariance of the current-current correlation function with respect to rotations of axis $\mathbf{u}_{z}$, one can establish the identity $\epsilon_{c} / 2-\Pi_{x x}^{33}=0$. It implies that the $A_{\mu}^{3^{2}}$ terms in the first- and second-order cumulants cancel each other, which ensures the $\mathrm{U}(1)$ gauge invariance. The only remaining correlator is the transverse spin susceptibility $\Pi_{00}^{11} \equiv \chi^{\perp}$. In order to express the effective action $S_{\text {eff }}[\mathbf{n}, \mathbf{L}]$ [Eq. (27)] in terms of $\mathbf{n}$ and $\mathbf{L}$ we use the relations (see Appendix $B$ )

$$
\begin{aligned}
\sum_{\nu=1,2} A_{\mu \mathbf{r}}^{\nu}{ }^{2} & =\zeta \frac{1}{4}\left(\partial_{\mu} \mathbf{n}_{\mathbf{r}}\right)^{2} \\
\sum_{\nu=1,2} A_{0 \mathbf{r}}^{\nu} l_{\mathbf{r}}^{\nu} & =\frac{i}{2}\left(\mathbf{n}_{\mathbf{r}} \wedge \partial_{\tau} \mathbf{n}_{\mathbf{r}}\right) \cdot \mathbf{L}_{\mathbf{r}}
\end{aligned}
$$

with $\zeta=1$ for $\mu=x, y$ and $\zeta=-1$ for $\mu=0$. Putting everything together, we obtain the effective action

$$
\begin{aligned}
S_{\mathrm{eff}}= & \frac{1}{2} \int_{0}^{\beta} d \tau \sum_{\mathbf{r}}\left[\frac{\chi^{\perp}}{4} \dot{\mathbf{n}}_{\mathbf{r}}^{2}+\frac{\epsilon_{c}}{8} \sum_{\mu=x, y}\left(\partial_{\mu} \mathbf{n}_{\mathbf{r}}\right)^{2}\right. \\
& \left.\left.+\Delta_{0}^{2}\left(\frac{2}{U}-\chi^{\perp}\right) \mathbf{L}_{\mathbf{r}}^{2}-i \Delta_{0} \chi^{\perp}\left(\mathbf{n}_{\mathbf{r}} \wedge \dot{\mathbf{n}}_{\mathbf{r}}\right) \cdot \mathbf{L}_{\mathbf{r}}\right] 37\right)
\end{aligned}
$$

where $\dot{\mathbf{n}}=\partial_{\tau} \mathbf{n}$. Integrating out the canting field with the constraint $\mathbf{L}_{\mathbf{r}} \perp \mathbf{n}_{\mathbf{r}}$, we eventually obtain a $\mathrm{NL} \sigma \mathrm{M}$ for the Néel field:

$$
S_{\mathrm{NL} \sigma \mathrm{M}}[\mathbf{n}]=\frac{\rho_{s}^{0}}{2} \int_{0}^{\beta} d \tau \int d^{2} r\left[\frac{1}{c^{2}} \dot{\mathbf{n}}_{\mathbf{r}}^{2}+\sum_{\mu=x, y}\left(\partial_{\mu} \mathbf{n}_{\mathbf{r}}\right)^{2}\right],
$$

where we have taken the continuum limit in real space. The bare spin stiffness $\rho_{s}^{0}$ and the spin-wave velocity $c$ are given by

$$
\rho_{s}^{0}=\frac{\epsilon_{c}}{8}, \quad c^{2}=\frac{\epsilon_{c}}{2}\left(\frac{1}{\chi^{\perp}}-\frac{U}{2}\right) .
$$

Eq. (38) must be supplemented with a cutoff $\Lambda$ in momentum space. In the strong-coupling limit, where $\mathrm{AF}$ fluctuations are due to local moments, the cutoff $\Lambda$ can be taken of the order of unity. In the weak-coupling limit, the Néel field is ill-defined at length-scales smaller than $\xi_{0} \sim t / \Delta_{0}$, since below $\xi_{0}$ "local" moments cannot be defined (see the discussion at the beginning of Sec. IIB). We therefore choose $\Lambda \sim \min \left(1,2 \Delta_{0} / c\right) ?^{71}$

For numerical computation of the spin-wave velocity and spin stiffness we use the expressions

$$
\begin{aligned}
\chi^{\perp} & =2 \Delta_{0}^{2} \int_{0}^{4 t} \frac{\rho_{0}(\epsilon) d \epsilon}{\left(\Delta_{0}^{2}+\epsilon^{2}\right)^{\frac{3}{2}}}, \\
\rho_{s}^{0} & =\frac{1}{4} \int_{0}^{4 t} \frac{\epsilon^{2} \rho_{0}(\epsilon) d \epsilon}{\sqrt{\Delta_{0}^{2}+\epsilon^{2}}} .
\end{aligned}
$$

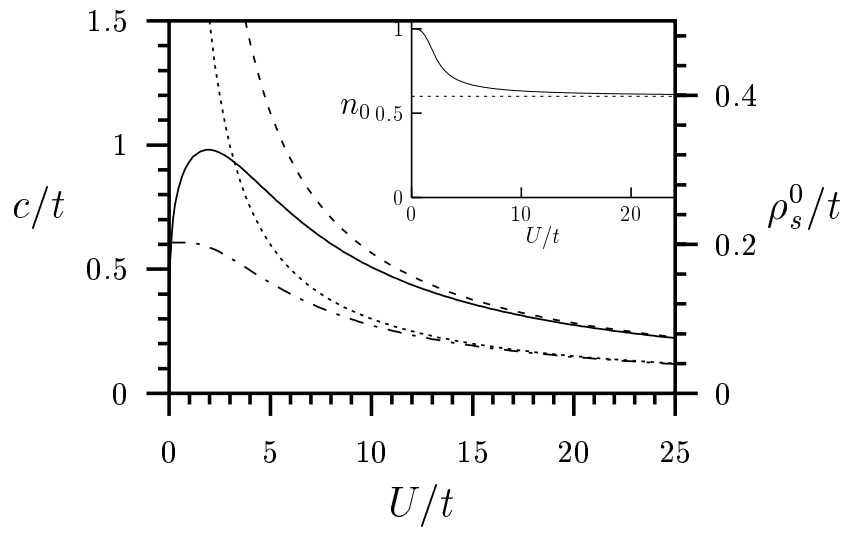

FIG. 1: Spin-wave velocity $c$ (solid line) and bare spin stiffness $\rho_{s}^{0}$ (dot-dashed line) vs $U$. For $U \gg 4 t$ we recover the results obtained from the Heisenberg model with $J=4 t^{2} / U$ (dashed line: spin-wave velocity, dotted line: bare spin stiffness). Inset: fraction of condensed bosons at $T=0.1-n_{0}$ is exponentially small at weak coupling $(U \ll 4 t)$, while $n_{0} \simeq 0.6$ for $U \gg 4 t$. $n_{0}$ determines the mean-value of the Néel field in the ground state $\left(\left\langle\mathbf{n}_{\mathbf{r}}\right\rangle=n_{0} \mathbf{u}_{z}\right)$ and the spectral weight of the Bogoliubov QP's (see Sec. IV).

$\rho_{0}$ is the density of states of free fermions on a square lattice. It can be expressed, using the complete elliptic integral of the first kind $K$, as $\rho_{0}(\epsilon)=\left(2 \pi^{2} t\right)^{-1} K[(1-$ $\left.\left.\epsilon^{2} / 16 t^{2}\right)^{1 / 2}\right]$ for $|\epsilon| \leq 4 t$. In the strong coupling limit we recover the results obtained from the Heisenberg model with an exchange coupling $J=4 t^{2} / U$ : the spin stiffness equals $J / 4$ and the spin-wave velocity $\sqrt{2} J$. At weak coupling, $c$ goes to zero like $2 \pi^{-1 / 2} t(U / t)^{\frac{1}{4}}$ and $\rho_{s}^{(0)} \sim t$. The factor $(U / t)^{\frac{1}{4}}$ is due to the Van-Hove singularities. The results are shown in Fig. 1.

The NL $\sigma \mathrm{M}$ defined by Eqs. (38 41) was first obtained by Schulz ${ }^{57}$ The value of the spin-wave velocity agrees with the result obtained from an RPA calculation about the zero-temperature AF HF state ${ }^{15.16,17}$

Note that we also expect a damping term with a characteristic frequency $\omega_{\mathrm{sf}}$ in the NL $\sigma \mathrm{M}$ action (38) at weak coupling. This term comes from the damping of spin fluctuations by fermion excitations which are gapless in the weak coupling limit (see Sec. IV]). It is missed in our approach since we expand around the zero-temperature AF state which has only gapped quasi-particle excitations. In the renormalized classical regime, this term is however negligible since $\omega_{\mathrm{sf}} \propto \xi^{-2} \rightarrow 0$ (critical slowing down) $\underline{13.45}$ 


\section{MAGNETIC PHASE DIAGRAM}

Let us recast the NL $\sigma \mathrm{M}$ action in a more usual form, by making use of the coupling constant $g=c / \rho_{s}^{0}$ :

$$
S_{\mathrm{NL} \sigma \mathrm{M}}[\mathbf{n}]=\frac{1}{2 g} \int_{0}^{\beta} d \tau \int d^{2} r\left[\frac{1}{c} \dot{\mathbf{n}}_{\mathbf{r}}^{2}+c \sum_{\mu=x, y}\left(\partial_{\mu} \mathbf{n}_{\mathbf{r}}\right)^{2}\right] .
$$

To solve the NL $\sigma \mathrm{M}$, we use a saddle-point approximation in the $\mathrm{CP}^{1}$ representation, ${ }^{11}$ which proves well suited for the computation of the fermion Green's function. In the $\mathrm{CP}^{1}$ representation, the Néel field is expressed in terms of two Schwinger bosons,

$$
\mathbf{n}_{\mathbf{r}}=\mathrm{z}_{\mathbf{r}}^{\dagger} \sigma \mathrm{z}_{\mathbf{r}}
$$

with $\mathrm{z}_{\mathbf{r}}=\left(z_{\mathbf{r} \uparrow}, z_{\mathbf{r} \downarrow}\right)^{T}$. The condition $\mathbf{n}_{\mathbf{r}}^{2}=1$ translates into $z_{\mathbf{r}}^{\dagger} z_{\mathbf{r}}=1$. The rotation matrix $R$ can be expressed as

$$
R_{\mathbf{r}}=\left(\begin{array}{cc}
z_{\mathbf{r} \uparrow} & -z_{\mathbf{r} \downarrow}^{\star} \\
z_{\mathbf{r} \downarrow} & z_{\mathbf{r} \uparrow}^{\star}
\end{array}\right)
$$

The U(1) gauge symmetry now manifests itself in the invariance of the $\mathbf{n}_{\mathbf{r}}$ vector and the relation (15) defining the rotation matrix under the transformation $z_{\mathbf{r}} \rightarrow$ $e^{i \alpha_{\mathbf{r}}} \mathrm{Z}_{\mathbf{r}}$.

The NL $\sigma \mathrm{M}$ expressed in terms of Schwinger bosons involves terms quadratic and quartic in $z_{\mathbf{r}}$. The latter turns out to be proportional to $A_{\mu \mathbf{r}}^{z}{ }^{2}$ with $A_{\mu \mathbf{r}}^{z}$ expressed in terms of Schwinger bosons [Eqs. (17) and (44)]. It is decoupled by an auxiliary field $a_{\mu \mathbf{r}}$. To handle the unimodularity condition $z_{\mathbf{r}}^{\dagger} z_{\mathbf{r}}=1$ one introduces Lagrange multipliers $\lambda_{\mathbf{r}}$ at each time and site. The partition function then becomes (see Ref. 11)

$$
\begin{aligned}
Z= & \int \mathcal{D}\left[\mathrm{z}, a_{\mu}, \lambda\right] e^{-S} \\
S= & \int_{0}^{\beta} d \tau \int d^{2} r\left[i \lambda_{\mathbf{r}}\left(\mathrm{z}_{\mathbf{r}}^{\dagger} \mathrm{z}_{\mathbf{r}}-1\right)+\frac{2}{g c}\left|\left(\partial_{\tau}-a_{0 \mathbf{r}}\right) \mathrm{z}_{\mathbf{r}}\right|^{2}\right. \\
& \left.+\frac{2 c}{g} \sum_{\mu=x, y}\left|\left(\partial_{\mu}-i a_{\mu \mathbf{r}}\right) \mathrm{z}_{\mathbf{r}}\right|^{2}\right]
\end{aligned}
$$

the $z_{\mathbf{r} \uparrow}$ and $z_{\mathbf{r} \downarrow}$ being now unconstrained bosonic fields. One then performs a saddle-point approximation over the $\lambda_{\mathbf{r}}$ and $a_{\mu \mathbf{r}}$ fields. When the $\mathrm{CP}^{1}$ representation is generalized to the $\mathrm{CP}^{N-1}$ representation by introducing $N$ different $z$ bosons, the approximation becomes exact in the limit $N \rightarrow \infty$.11 Within the ansatz of a uniform static saddle-point solution $i \lambda_{\mathbf{r}}=2 \mathrm{~m}^{2} / \mathrm{gc}$ and $a_{\mu \mathbf{r}}=0$, the propagator can be read off from (46):

$$
\begin{aligned}
& -\left\langle z_{\mathbf{q} \omega_{\nu} \sigma} z_{\mathbf{q}^{\prime} \omega_{\nu}^{\prime} \sigma^{\prime}}^{\star}\right\rangle=\delta_{\mathbf{q}, \mathbf{q}^{\prime}} \delta_{\omega_{\nu}, \omega_{\nu}^{\prime}} \delta_{\sigma, \sigma^{\prime}} \mathcal{D}_{\sigma}\left(\mathbf{q}, \omega_{\nu}\right) \\
& \mathcal{D}_{\sigma}\left(\mathbf{q}, \omega_{\nu}\right)=\frac{-g c}{2\left(\omega_{\nu}^{2}+\omega_{\mathbf{q}}^{2}\right)}-\beta \mathcal{N} n_{0} \delta_{\sigma, \uparrow} \delta_{\omega_{\nu}, 0} \delta_{\mathbf{q}, \mathbf{0}}, \\
& \omega_{\mathbf{q}}=\sqrt{c^{2} \mathbf{q}^{2}+m^{2}}
\end{aligned}
$$

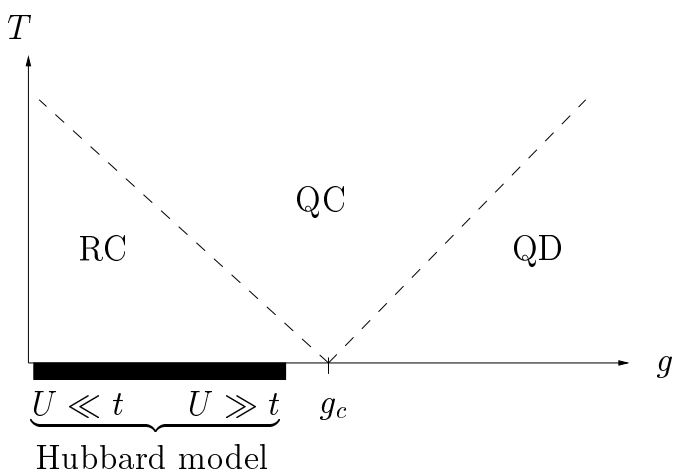

FIG. 2: Phase diagram of the NL $\sigma \mathrm{M}$ derived from a saddlepoint approximation in the $\mathrm{CP}^{1}$ representation. At $T=0$, there is long-range order when the coupling constant $g<$ $g_{c}=4 \pi / \Lambda$. The three finite-temperature regimes correspond to "renormalized classical" (RC), "quantum critical" (QC) and "quantum disordered" (QD). ${ }^{28.72}$ The ground state of the 2D half-filled Hubbard model is ordered for any value of the Coulomb repulsion $U$. At finite temperature, there are strong AF fluctuations with an exponentially large correlation length $\xi \gg 1$ (RC regime).

where $\mathcal{N}$ is the number of lattice sites. The saddle-point equation for the Lagrange multiplier $m^{2}$ reads

$$
\frac{1}{\beta} \sum_{\omega_{\nu}} \int_{|\mathbf{q}|<\Lambda} \frac{g c}{\omega_{\nu}^{2}+\omega_{\mathbf{q}}^{2}}+n_{0}=1 .
$$

In Eqs. (47:50), we have allowed for a Bose condensation of the Schwinger bosons in the mode $\mathbf{q}=\mathbf{0}$, with $n_{0}=\frac{1}{\mathcal{N} \beta}\left\langle\mathrm{z}^{\dagger}\left(\mathbf{q}=\mathbf{0}, \omega_{\nu}=0\right) \mathrm{z}\left(\mathbf{q}=\mathbf{0}, \omega_{\nu}=0\right)\right\rangle$ the fraction of condensed bosons. Bose condensation signals the appearance of AF long-range order: $\left\langle\mathbf{n}_{\mathbf{r}}\right\rangle=n_{0} \mathbf{u}_{z}$. Knowing the propagator of the $z$ field, one can then calculate the spin-spin correlation function using Eq. (43). The AF correlation length $\xi$ is related to the mass $m$ of the bosonic propagator $\mathcal{D}$ via $m=c / 2 \xi .^{11} m$ vanishes whenever the fraction of the condensed bosons is finite.

At zero temperature, the solution of the saddle-point equation (50) shows that the NL $\sigma \mathrm{M}$ is ordered at small $g\left(m=0\right.$ and $\left.n_{0}>0\right)$ and disordered by quantum fluctuations at large $g\left(m>0\right.$ and $\left.n_{0}=0\right)$. The two regimes are separated by a quantum-critical point at $g_{c}=4 \pi / \Lambda$. In the ordered phase $\left(g \leq g_{c}\right)$, the fraction of condensed bosons is $n_{0}=1-g / g_{c}$.

The condition of zero-temperature long-range order is satisfied in the NL $\sigma \mathrm{M}$ derived from the half-filled Hubbard model (Fig. 2). For $U \ll 4 t, g / g_{c} \sim e^{-2 \pi \sqrt{t / U}}$ is exponentially small. For $U \gg 4 t, \rho_{s}^{0} \simeq J / 4$ and $c \Lambda \simeq \sqrt{2} J$, so that $g / g_{c} \simeq \sqrt{2} / \pi<1$. Notice that setting the cutoff to a higher value at strong coupling would lead us into the quantum disordered regime. However, our choice is consistent with results obtained by mapping the Hubbard model at strong coupling onto the Heisenberg model. It is known, both from numerical and analytical work, that the $2 \mathrm{D}$ quantum Heisenberg model on a square lattice is ordered at zero temperature ${ }^{73}$ 


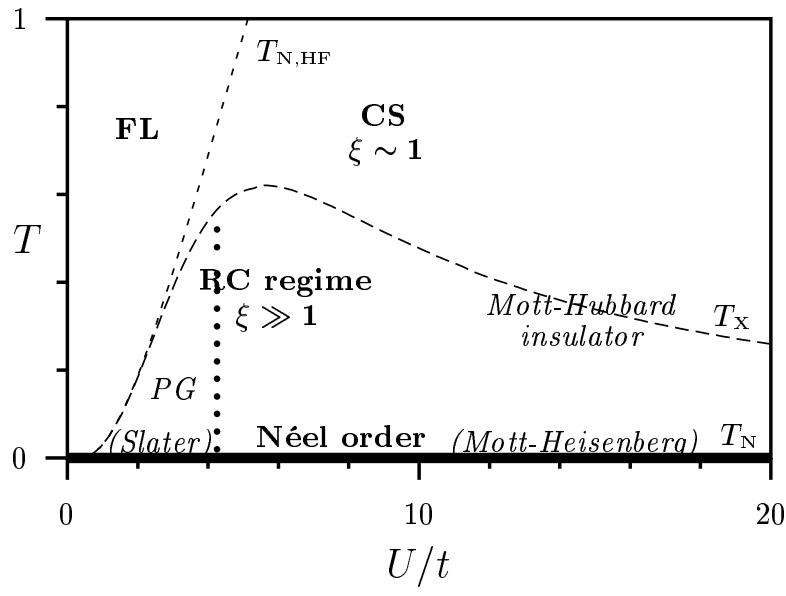

FIG. 3: Phase diagram of the 2D half-filled Hubbard model. $T \gtrsim T_{\mathrm{N}}^{\mathrm{HF}}:$ Fermi liquid (FL) phase; $T_{\mathrm{X}} \lesssim T \lesssim T_{\mathrm{N}}^{\mathrm{HF}}:$ local moments with no AF short-range order (Curie spins, $\xi \sim 1$ ); $T=0$ : Slater $(U \ll 4 t)$ and Mott-Heisenberg $(U \gg 4 t)$ antiferromagnets. At finite temperature, there is a pseudogap phase $(U \ll 4 t)$ and a Mott-Hubbard insulator $(U \gg 4 t)$ separated by a metal-insulator transition (dotted line) defined by the vanishing of the tunneling density of states $\rho(\omega=0)$ at zero energy (Sec. IV). All lines, except $T_{\mathrm{N}}=0$ (thick solid line), are crossover lines. The NL $\sigma \mathrm{M}$ description is valid below $T_{X}$ (RC regime). [From Ref. 56.]

Fig. 1 shows the fraction of condensed bosons as a function of $U$. For this, and subsequent, numerical calculations we use a smooth cutoff, i.e. $\int_{|\mathbf{q}|<\Lambda} \rightarrow$ $\int_{\mathbf{q}} \frac{e^{-|\mathbf{q}| \xi_{0}}-e^{-q_{0} \xi_{0}}}{1-e^{-q_{0} \xi_{0}}}$. In contrast to a hard cutoff, this procedure prevents artificial features in the fermion spectral function and in the density of states. The parameter $q_{0}$ is adjusted so as to reproduce in the strong-coupling limit $(U \gg 4 t)$ the result $\left|\left\langle\mathbf{n}_{\mathbf{r}}\right\rangle\right|=n_{0} \simeq 0.6$ obtained from the Heisenberg model ${ }^{73}$ While the value of $n_{0}$ for $U \ll 4 t$ and $U \gg 4 t$ does not depend on $\xi_{0}$, the behavior at intermediate coupling is strongly cutoff dependent.

At finite temperature, the AF long-range order is suppressed $\left(n_{0}=0, m>0\right)$, in agreement with the MerminWagner theorem. For systems that exhibit AF long-range order at $T=0$, the correlation length remains nevertheless exponentially large at low temperature [renormalized classical regime, see Fig. (2)]. From Eq. (50), we deduce

$$
\xi=\frac{c}{2 m}, \quad m=T e^{-\frac{2 \pi \rho_{s}}{T}},
$$

where $\rho_{s}=\rho_{s}^{0}\left(1-g / g_{c}\right)$ is the zero-temperature spin stiffness. The mass $m$ of the bosonic propagator being much smaller than the temperature, the dominant fluctuations are classical.

Let us now discuss the limits of validity of the NL $\sigma \mathrm{M}$. The derivation of the NL $\sigma \mathrm{M}$ is based on the assumption that the dominant low-energy fluctuations are transverse spin waves with a large correlation length. The condition $T \ll T_{\mathrm{N}}^{\mathrm{HF}}$ ensures that amplitude fluctuations of the AF order parameter are frozen at low energy. One should also verify that the computation of $\xi$ within the $\mathrm{NL} \sigma \mathrm{M}$ is consistent with the assumption of AF short-range order, i.e. $\xi \gg \Lambda^{-1}$ or, equivalently, $m \ll c \Lambda / 2$. We define $T^{\prime}$ as the solution of the equation $m \sim c \Lambda / 2$ obtained from Eq. (50). Then, the domain of validity of the NL $\sigma \mathrm{M}$ is given by $T \ll T_{\mathrm{X}} \sim \min \left(T_{\mathrm{N}}^{\mathrm{HF}}, T^{\prime}\right)$. At weak coupling, $T_{X} \sim T_{\mathrm{N}}^{\mathrm{HF}}$, while $T_{X} \sim T^{\prime} \sim J$ at strong coupling. The crossover temperature $T_{\mathrm{X}}$ displayed in Fig. 3 is a smooth interpolation between $T_{\mathrm{N}}^{\mathrm{HF}}$ and $T^{\prime}{ }^{74}$

The phase diagram is shown in Fig. 3. Above $T_{\mathrm{N}}^{\mathrm{HF}}$, spin fluctuations are not important and we expect a Fermi liquid behavior. Between $T_{\mathrm{N}}^{\mathrm{HF}}$ and $T_{\mathrm{X}}$ (a regime which exists only in the strong-coupling limit), local moments form but with no AF short-range order (Curie spins: $\xi \sim 1)$. Below $T_{\mathrm{X}}$, the system enters a renormalized classical regime of spin fluctuations where the AF correlation length becomes exponentially large [Eq. (51)]. AF long-range order sets in at $T_{\mathrm{N}}=0$. Although there is a smooth evolution of the magnetic properties as a function of $U$, the physics is quite different for $U \ll 4 t$ and $U \gg 4 t$. This will be shown in Sec. IV by studying the fermion spectral properties. The main conclusions are shown in Fig. 3. At zero temperature the system is an antiferromagnet, which evolves from a Slater to a Mott-Heisenberg behavior as $U$ increases. At finite temperature there is a pseudogap phase for $U \ll 4 t$ and a Mott-Hubbard insulator for $U \gg 4 t$. These two regimes are separated by a (finite-temperature) metal-insulator transition (dotted line in Fig. 3) defined by the vanishing of the tunneling density of states $\rho(\omega=0)$ at zero energy.

\section{FERMION SPECTRAL PROPERTIES}

In this section, we study the influence of the longwavelength spin fluctuations on the fermion spectral properties. The fermionic Green's function $-\left\langle\Psi_{\mathbf{r}_{1} \tau_{1}} \Psi_{\mathbf{r}_{2} \tau_{2}}^{\dagger}\right\rangle$, written here as a $2 \times 2$ matrix in spin space, can easily be related to the pseudo-fermions by use of the relation $\Psi_{\mathbf{r}}=R_{\mathbf{r}} \Phi_{\mathbf{r}}$ :

$$
\mathcal{G}(1,2)=-\left\langle R_{1} \Phi_{1} \Phi_{2}^{\dagger} R_{2}^{\dagger}\right\rangle
$$

Here we use the shorthand notations $1 \equiv\left(\mathbf{r}_{1}, \tau_{1}\right)$ and $2 \equiv$ $\left(\mathbf{r}_{2}, \tau_{2}\right)$. The averaging in the above expression should be performed with respect to the action $S_{\mathrm{HF}}[\Phi]+S^{\prime}[\mathrm{z}, \Phi, \mathbf{L}]$ obtained in Sec. IIB from the second-order expansion in $\mathbf{L}$ and $\partial_{\mu} \mathbf{n}$. $S^{\prime}$ stands for the sum of the perturbative corrections $S_{\mathrm{p}}, S_{\mathrm{d}}, S_{\mathrm{l}}, S_{\mathrm{1}^{2}}$ defined in (21 24). Integrating first the pseudo-fermions, we can write the propagator as

$$
\begin{aligned}
\mathcal{G}(1,2) & =\frac{1}{Z} \int \mathcal{D}[\mathrm{z}] e^{-S_{\mathrm{NL} \sigma \mathrm{M}}[\mathrm{z}]} R_{1} \mathcal{G}(1,2 \mid \mathrm{z}) R_{2}^{\dagger}, \\
Z & =\int \mathcal{D}[\mathrm{z}] e^{-S_{\mathrm{NL} \sigma \mathrm{M}}[z]}
\end{aligned}
$$


where $\mathcal{G}(1,2 \mid \mathrm{z})$ is the pseudo-fermion propagator calculated for a given configuration of the bosonic field $\mathrm{z}$ :

$$
\mathcal{G}(1,2 \mid \mathrm{z})=-\frac{\int \mathcal{D}[\Phi, \mathbf{L}] \phi_{1} \phi_{2}^{\star} e^{-S_{\mathrm{HF}}[\Phi]-S^{\prime}[\mathrm{z}, \Phi, \mathbf{L}]}}{\int \mathcal{D}[\Phi, \mathbf{L}] e^{-S_{\mathrm{HF}}[\Phi]-S^{\prime}[\mathbf{z}, \Phi, \mathbf{L}]}} .
$$

The action $S_{\mathrm{HF}}[\Phi]+S^{\prime}[\mathrm{z}, \Phi, \mathbf{L}]$ describes HF fermions interacting with spin fluctuations via the action $S^{\prime}$. Since the HF pseudo-fermions are gapped, we expect a perturbative expansion in $S^{\prime}$ to be well-behaved. To leading order, $\mathcal{G}(1,2 \mid \mathrm{z})=\mathcal{G}^{\mathrm{HF}}(1,2)$ and the fermion Green's function simplifies into

$$
\mathcal{G}_{\sigma_{1} \sigma_{2}}(1,2)=\sum_{\alpha_{1}, \alpha_{2}} \mathcal{G}_{\alpha_{1} \alpha_{2}}^{\mathrm{HF}}(1,2)\left\langle\left(R_{1}\right)_{\sigma_{1} \alpha_{1}}\left(R_{2}\right)_{\sigma_{2} \alpha_{2}}^{\star}\right\rangle,
$$

where the product of rotation matrices is averaged with the NL $\sigma \mathrm{M}$ action. This approximation neglects the effect of spin fluctuations on the propagation of pseudofermions. Their influence on the propagation of fermions is implemented only through the decomposition of the fermion into a boson and a pseudo-fermion.

Using the Schwinger boson propagator derived in Sec. III [Eqs. [4749)], we have

$$
\begin{aligned}
\left\langle\left(R_{1}\right)_{\sigma_{1} \alpha_{1}}\left(R_{2}\right)_{\sigma_{2} \alpha_{2}}^{\star}\right\rangle= & -\delta_{\sigma_{1}, \sigma_{2}} \delta_{\alpha_{1}, \alpha_{2}} \\
& \times\left(\overline{\mathcal{D}}(1,2)-\delta_{\sigma_{1}, \alpha_{1}} n_{0}\right),
\end{aligned}
$$

where $\overline{\mathcal{D}}$ is the non-condensed part of $\mathcal{D}_{\sigma}$. Using this expression in Eq. (56) we finally obtain for the fermion Green's function:

$$
\begin{aligned}
-\left\langle\psi_{\mathbf{r} \tau \sigma} \psi_{\mathbf{r}^{\prime} \tau^{\prime} \sigma^{\prime}}^{\star}\right\rangle & =\delta_{\sigma, \sigma^{\prime}} \mathcal{G}_{\sigma}\left(\mathbf{r}, \mathbf{r}^{\prime}, \tau-\tau^{\prime}\right), \\
\mathcal{G}_{\sigma}\left(\mathbf{k}, \mathbf{k}^{\prime}, \omega\right) & =-\frac{2 \delta_{\mathbf{k}, \mathbf{k}^{\prime}}}{\beta} \sum_{\omega_{\nu}} \int_{\mathbf{q}} \mathcal{G}_{\sigma}^{\mathrm{HF}}\left(\mathbf{k}-\mathbf{q}, \mathbf{k}-\mathbf{q}, \omega-\omega_{\nu}\right) \overline{\mathcal{D}}\left(\mathbf{q}, \omega_{\nu}\right)+n_{0} \mathcal{G}_{\sigma}^{\mathrm{HF}}\left(\mathbf{k}, \mathbf{k}^{\prime}, \omega\right) .
\end{aligned}
$$

Since $n_{0}$ vanishes at finite temperature, the fermion Green's function is spin-rotation and translation invariant in the absence of AF long-range order. We show below that the first term of the rhs of (59) corresponds to incoherent excitations. At zero temperature, the last term of (59) describes Bogoliubov QP's carrying a total spectral weight $n_{0}$.

To study in detail the fermion excitations, we consider the spectral function $\mathcal{A}(\mathbf{k}, \omega)=-\pi^{-1} \operatorname{Im} \mathcal{G}_{\sigma}(\mathbf{k}, \mathbf{k}, i \omega \rightarrow$ $\omega+i 0^{+}$) and the tunneling density of states (DOS) $\rho(\omega)=\int d \omega \mathcal{A}(\mathbf{k}, \omega)$. Performing the summation over bosonic Matsubara frequencies in Eq. (59) we obtain

$$
\begin{aligned}
\mathcal{A}(\mathbf{k}, \omega)= & \mathcal{A}_{\mathrm{inc}}(\mathbf{k}, \omega)+n_{0} \mathcal{A}_{\mathrm{HF}}(\mathbf{k}, \omega), \\
\mathcal{A}_{\mathrm{inc}}(\mathbf{k}, \omega)= & \int_{\mathbf{q}} \frac{g c}{2 \omega_{q}}\left\{\left[n_{B}\left(\omega_{\mathbf{q}}\right)+n_{F}\left(-E_{\mathbf{k}-\mathbf{q}}\right)\right]\left[u_{\mathbf{k}-\mathbf{q}}^{2} \delta\left(\omega-\omega_{\mathbf{q}}-E_{\mathbf{k}-\mathbf{q}}\right)+v_{\mathbf{k}-\mathbf{q}}^{2} \delta\left(\omega+\omega_{\mathbf{q}}+E_{\mathbf{k}-\mathbf{q}}\right)\right]\right. \\
& \left.+\left[n_{B}\left(\omega_{\mathbf{q}}\right)+n_{F}\left(E_{\mathbf{k}-\mathbf{q}}\right)\right]\left[u_{\mathbf{k}-\mathbf{q}}^{2} \delta\left(\omega+\omega_{\mathbf{q}}-E_{\mathbf{k}-\mathbf{q}}\right)+v_{\mathbf{k}-\mathbf{q}}^{2} \delta\left(\omega-\omega_{\mathbf{q}}+E_{\mathbf{k}-\mathbf{q}}\right)\right]\right\},
\end{aligned}
$$

where $n_{F}(\omega)$ and $n_{B}(\omega)$ are the usual Fermi and Bose occupation numbers $\left(e^{\beta \omega} \pm 1\right)^{-1}$, and $\mathcal{A}_{\mathrm{HF}}$ the HF spectral function:

$$
\begin{aligned}
& \mathcal{A}_{\mathrm{HF}}(\mathbf{k}, \omega)=u_{\mathbf{k}}^{2} \delta\left(\omega-E_{\mathbf{k}}\right)+v_{\mathbf{k}}^{2} \delta\left(\omega+E_{\mathbf{k}}\right), \\
& u_{\mathbf{k}}^{2}=\frac{1}{2}\left(1+\frac{\epsilon_{\mathbf{k}}}{E_{\mathbf{k}}}\right), \quad v_{\mathbf{k}}^{2}=\frac{1}{2}\left(1-\frac{\epsilon_{\mathbf{k}}}{E_{\mathbf{k}}}\right) .
\end{aligned}
$$

One can check that the spectral function $\mathcal{A}(\mathbf{k}, \omega)$ is normalized to unity. From Eqs. (60,61) we deduce

$$
\begin{aligned}
\int d \omega \mathcal{A}(\mathbf{k}, \omega) & =\int_{|\mathbf{q}|<\Lambda} \frac{g c}{\omega_{\mathbf{q}}}\left(n_{B}\left(\omega_{\mathbf{q}}\right)+\frac{1}{2}\right)+n_{0} \\
& =1
\end{aligned}
$$

where the second equality is obtained by using $\left\langle z_{\mathbf{r}}^{\dagger} z_{\mathbf{r}}\right\rangle=1$ [Eq. (50)]. From Eqs. (60:63), we obtain

$$
\rho(\omega)=\rho_{\mathrm{inc}}(\omega)+n_{0} \rho_{\mathrm{HF}}(\omega),
$$

$$
\begin{aligned}
\rho_{\mathrm{inc}}(\omega)= & \rho_{\mathrm{inc}}^{>}(\omega)+\rho_{\mathrm{inc}}^{>}(-\omega), \\
\rho_{\mathrm{inc}}^{>}(\omega)= & \frac{g}{4 \pi c} \int_{m}^{c \Lambda} d \omega^{\prime}\left[n_{B}\left(\omega^{\prime}\right) \rho_{\mathrm{HF}}\left(\omega+\omega^{\prime}\right) \theta\left(\omega+\omega^{\prime}\right)\right. \\
& \left.+\left(n_{B}\left(\omega^{\prime}\right)+1\right) \rho_{\mathrm{HF}}\left(\omega-\omega^{\prime}\right) \theta\left(\omega-\omega^{\prime}\right)\right], \quad(67)
\end{aligned}
$$

where

$$
\rho_{\mathrm{HF}}(\omega)=\theta\left(\omega^{2}-\Delta_{0}^{2}\right) \frac{|\omega|}{\sqrt{\omega^{2}-\Delta_{0}^{2}}} \rho_{0}\left(\sqrt{\omega^{2}-\Delta_{0}^{2}}\right)
$$

is the HF DOS and $\theta$ the step function. We have approximated the Fermi occupation numbers by their zerotemperature limit, which is valid for $T \ll T_{\mathrm{N}}^{\mathrm{HF}}$. 


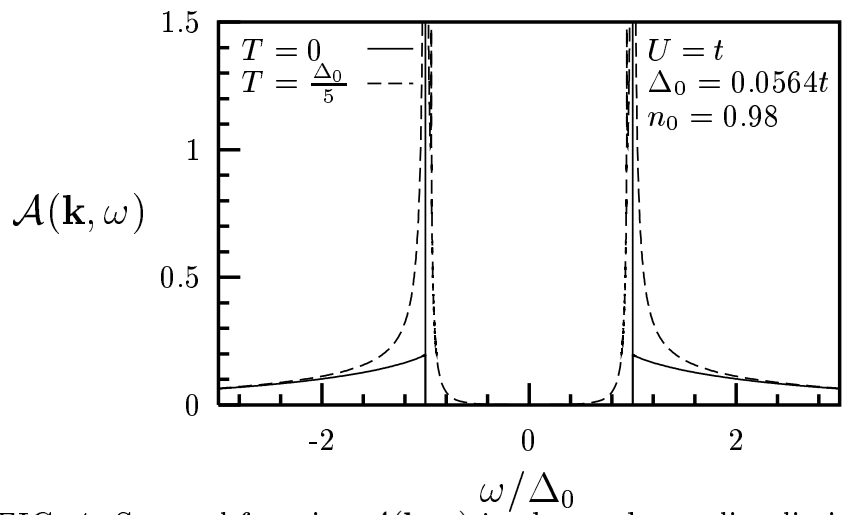

FIG. 4: Spectral function $\mathcal{A}(\mathbf{k}, \omega)$ in the weak-coupling limit $U=t$ for $T=0$ (Slater antiferromagnet) and $T=\Delta_{0} / 5$ (pseudogap phase). $\mathbf{k}=(\pi / 2, \pi / 2)$. The vertical lines represent Dirac peaks of weight $n_{0} / 2$ (Bogoliubov QP's). At finite temperature, precursors of the zero-temperature Bogoliubov QP's show up as peaks of width $\sim T$ at $\pm E_{\mathbf{k}}$. At low energy (and $T>0$ ), we observe a pseudogap with an exponentially small spectral weight at $\omega=0$. Energies are measured in units of $t$. [From Ref. 56.]

\section{A. $T=0$ : Slater vs Mott-Heisenberg antiferromagnetism}

At zero temperature, the incoherent part of the spectral function [Eq. [61)] can be simplified. All the occupation factors vanish, except fermionic factors at negative energies which are equal to 1 , so that

$$
\begin{array}{r}
\mathcal{A}_{\text {inc }}(\mathbf{k}, \omega)=\int_{\mathbf{q}} \frac{g c}{2 \omega_{q}}\left[u_{\mathbf{k}-\mathbf{q}}^{2} \delta\left(\omega-\omega_{\mathbf{q}}-E_{\mathbf{k}-\mathbf{q}}\right)\right. \\
\left.+v_{\mathbf{k}-\mathbf{q}}^{2} \delta\left(\omega+\omega_{\mathbf{q}}+E_{\mathbf{k}-\mathbf{q}}\right)\right] .
\end{array}
$$

In the same way, we obtain for the DOS

$$
\rho_{\mathrm{inc}}^{>}(\omega)=\frac{g}{4 \pi c} \int_{0}^{c \Lambda} d \omega^{\prime} \rho_{\mathrm{HF}}\left(\omega-\omega^{\prime}\right) \theta\left(\omega-\omega^{\prime}\right) .
$$

In Figs. 4.5 we show the spectral function at the $\mathbf{k}=(\pi / 2, \pi / 2)$ point of the non-interacting Fermi surface at weak $(U=t)$ and strong $(U=12 t)$ coupling. The spectral function $\mathcal{A}(\mathbf{k}, \omega)$ exhibits a gap $2 \Delta_{0}$, which is a consequence of AF long-range order. There are welldefined Bogoliubov QP's with excitation energy $\pm E_{\mathbf{k}}$, as in HF theory, but their spectral weight is reduced by a factor $n_{0}<1$ because of quantum spin fluctuations. The remaining weight $\left(1-n_{0}\right)$ is carried by an incoherent excitation background at higher energy $\left(|\omega|>E_{\mathbf{k}}\right)$.

There are important differences between the weak $(U \ll 4 t)$ and strong $(U \gg 4 t)$ coupling regimes. First, the AF gap $2 \Delta_{0} \sim t e^{-2 \pi \sqrt{t / U}}$ is exponentially small at weak coupling, while it tends to $U$ for $U \gg 4 t$. Second, the Bogoliubov QP's carry most of the spectral weight in the weak-coupling regime, since $g / g_{c}=1-n_{0}$ is exponentially small when $U \ll 4 t$. As $U$ increases, spectral weight is transferred from the Bogoliubov QP's to the incoherent excitation background, and at strong coupling

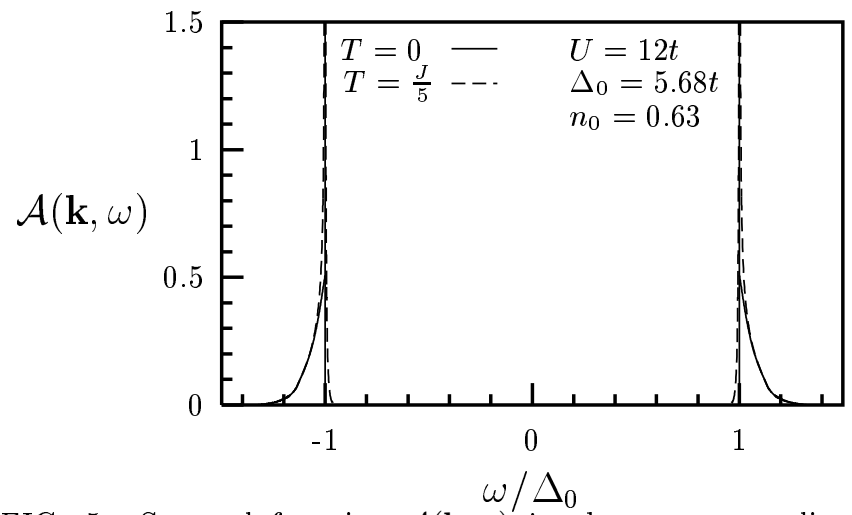

FIG. 5: Spectral function $\mathcal{A}(\mathbf{k}, \omega)$ in the strong-coupling regime $U=12 t$ for $T=0$ (Mott-Heisenberg antiferromagnet) and $T=J / 5$ (Mott-Hubbard insulator). At $T=0$, when $U$ increases, spectral weight is transferred from the Bogoliubov $\mathrm{QP}$ peaks to the incoherent excitation background. [Note the difference in the energy scale, which is fixed by $\Delta_{0}$, between Figs. 4] and 5] [From Ref. 56.]

$(U \gg 4 t)$ the incoherent excitation background carries a significant fraction of the total spectral weight (i.e. $n_{0}$ and $1-n_{0}$ are of the same order). Third, the energy range of the incoherent excitation background depends on the value of $U$. From Eq. (69) we see that it extends from $E_{\mathbf{k}}$ to $\sim \sqrt{E_{\mathbf{k}}^{2}+16 t^{2} \Lambda^{2}}+c \Lambda$. At weak coupling, the upper limit turns out to be of order $\Delta_{0}$ (for $\mathbf{k}$ lying on the non-interacting Fermi surface). Thus, the energy range of the incoherent excitation background remains very small with respect to the dispersion of the Bogoliubov QP energy $E_{\mathbf{k}}$, which is of order $t$ when $\Delta_{0} \ll t$. At strong coupling, the incoherent excitation background above $E_{\mathbf{k}} \sim U / 2$ extends over a range of order $J$. This energy range is of the same order of magnitude as the dispersion of the Bogoliubov QP energy, which is also of order $J$ when $U \gg 4 t$ [as can be seen from the expansion $\left.E_{\mathbf{k}} \simeq U / 2+J\left(\cos k_{x}+\cos k_{y}\right)^{2}\right]$.

In Figs. 67 we compare the zero-temperature DOS $\rho(\omega)$ and the non-interacting DOS $\rho_{0}(\omega)$. At weak coupling $(U=t), \rho(\omega)$ is similar to the HF result, with no visible effect of the incoherent excitation background. $\rho(\omega)$ differs from $\rho_{0}(\omega)$ mainly at low energy, due to the (small) AF gap 2 $\Delta_{0}$. At strong coupling $(U=12 t), \rho(\omega)$ differs strongly from $\rho_{0}(\omega)$, due to an AF gap $2 \Delta_{0} \sim U$ exceeding the non-interacting bandwidth. There is also a significant difference between $\rho(\omega)$ and $\rho_{\mathrm{HF}}(\omega)$, which results from the incoherent excitation background.

The spectral function $\mathcal{A}(\mathbf{k}, \omega)$ and the DOS $\rho(\omega)$ are typical of a Slater antiferromagnet at weak coupling and of a Mott-Heisenberg antiferromagnet at strong coupling. As shown in the next section, Slater and Mott-Heisenberg antiferromagnets behave very differently at finite temperature. 


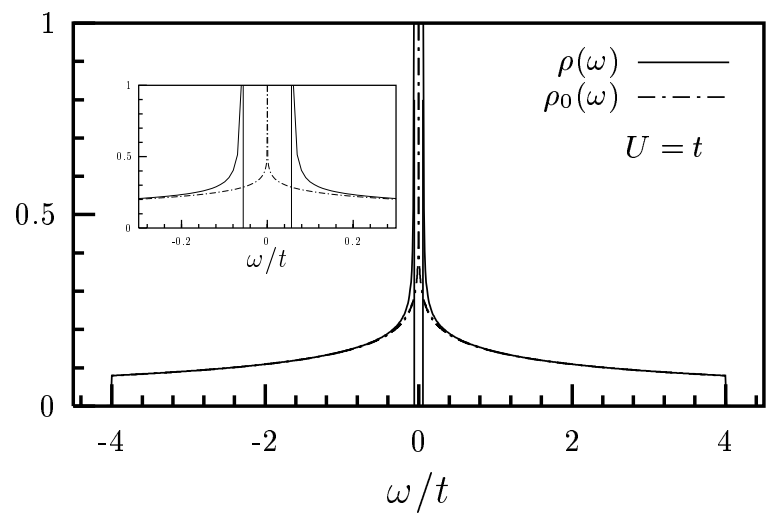

FIG. 6: Zero-temperature DOS $\rho(\omega)$ at weak coupling: $U=t$ (Slater antiferromagnet). $\rho(\omega)$ differs from the free-fermion DOS $\rho_{0}(\omega)$ only at low energy due to the opening of the AF gap $2 \Delta_{0}$ (see inset). Since the incoherent excitation background carries a negligible fraction of the total spectral weight, there is no noticeable difference between $\rho(\omega)$ and the HF DOS $\rho_{\mathrm{HF}}(\omega)$ (not shown in the figure).

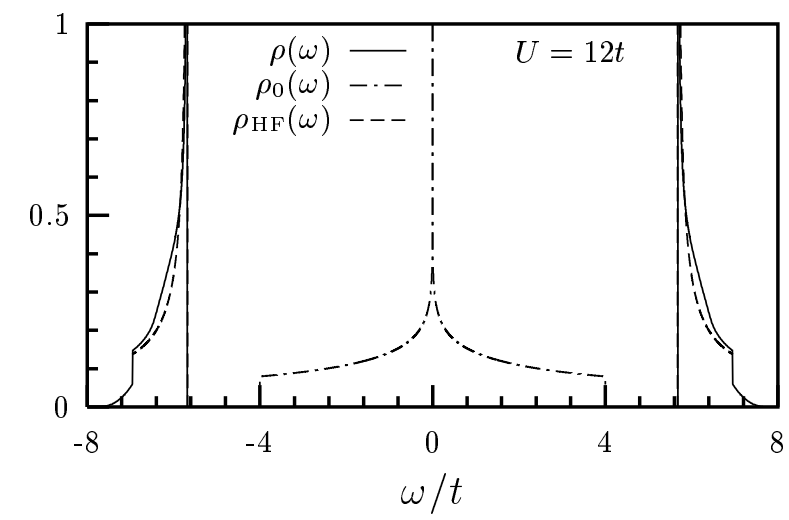

FIG. 7: Same as Fig. [6] but at strong coupling: $U=12 t$ (Mott-Heisenberg antiferromagnet). $\quad \rho(\omega)$ differs strongly from the non-interacting DOS $\rho_{0}(\omega)$, as the AF gap exceeds the non-interacting bandwidth. It also differs from the HF $\operatorname{DOS} \rho_{\mathrm{HF}}(\omega)$ due to the incoherent excitation background carrying a significant fraction of the total spectral weight.

\section{B. $T>0$ : pseudogap vs Mott-Hubbard gap}

At finite temperature, $n_{0}$ vanishes and $\mathcal{A}=\mathcal{A}_{\text {inc }}$. The result of the numerical calculation for $U=t$ and $U=12 t$ for $\mathbf{k}=(\pi / 2, \pi / 2)$ is shown in Figs. 4 and $\mathbf{5}$. $\mathcal{A}(\mathbf{k}, \omega)$ exhibits broadened peaks of width $T$ at the HF QP energy $\pm E_{\mathbf{k}}$. These peaks are incoherent precursors of the zero-temperature Bogoliubov QP peaks. The zerotemperature AF gap is partially filled at strong coupling and transforms into a pseudogap in the weak-coupling regime. At higher energy $\left(|\omega| \gtrsim E_{\mathbf{k}}\right)$, a roughly featureless incoherent excitation background is observed.

\section{Precursors of Bogoliubov QP's}

At finite temperature, the coherent part of the spectral function disappears. However, sharp peaks are still observed at the HF energy $\pm E_{\mathbf{k}}$. To study the peak at $E_{\mathbf{k}}$, let us perform a few approximations on the finitetemperature spectral function (61). First, at positive energies, almost all the spectral weight comes from the terms proportional to $u_{\mathbf{k}-\mathbf{q}}^{2}$ in (61) (except at energies close to zero), whose sum will be denoted by $\mathcal{A}^{>}$. Second, we replace the Fermi occupation number by the step function, given that the temperature is small compared to $E_{\mathbf{k}}$. Regrouping terms containing the Bose occupation numbers we obtain

$$
\begin{aligned}
\mathcal{A}^{>}(\mathbf{k}, \omega)= & \mathcal{A}_{\mathrm{bg}}^{>}(\mathbf{k}, \omega)+\mathcal{A}_{\text {peak }}^{>}(\mathbf{k}, \omega), \\
\mathcal{A}_{\text {peak }}^{>}(\mathbf{k}, \omega)= & \int_{\mathbf{q}} \frac{g c}{2 \omega_{\mathbf{q}}} n_{B}\left(\omega_{\mathbf{q}}\right) u_{\mathbf{k}-\mathbf{q}}^{2}\left[\delta\left(\omega-\omega_{\mathbf{q}}-E_{\mathbf{k}-\mathbf{q}}\right)\right. \\
& \left.+\delta\left(\omega+\omega_{\mathbf{q}}-E_{\mathbf{k}-\mathbf{q}}\right)\right] .
\end{aligned}
$$

$\mathcal{A}_{\mathrm{bg}}^{>}$has the same expression as the incoherent excitation background term (69) at zero temperature. It thus describes a temperature-independent incoherent excitation background at energies above $E_{\mathbf{k}}$. $\mathcal{A}_{\text {peak }}^{>}$gives rise to the peak at the HF energy $E_{\mathbf{k}}$. To see this, let us put it into a more explicit form. Because of the bosonic occupation numbers, the sum over $\mathbf{q}$ in (72) is dominated by wavevectors satisfying $\omega_{\mathbf{q}} \lesssim T$ or, equivalently, $|\mathbf{q}| \lesssim T / c$. For $T \ll T_{X}, T / c \ll 1$ and we can neglect the $\mathbf{q}$ dependence of $E_{\mathbf{k}-\mathbf{q}}$ and $u_{\mathbf{k}-\mathbf{q}}^{2}$. The integrand then becomes isotropic, and one can use $\int_{\mathbf{q}} \frac{c^{2}}{\omega_{\mathbf{q}}}=\int_{m}^{c \Lambda} \frac{d \omega_{\mathbf{q}}}{2 \pi}$. The result is

$$
\mathcal{A}_{\text {peak }}^{>}(\mathbf{k}, \omega)=u_{\mathbf{k}}^{2} \frac{g}{4 \pi c} n_{B}\left(\left|\omega-E_{\mathbf{k}}\right|\right)
$$

for $\left|\omega-E_{\mathbf{k}}\right|>m$, and vanishes for $\left|\omega-E_{\mathbf{k}}\right|<m$. For $m<\left|\omega-E_{\mathbf{k}}\right| \ll T, \mathcal{A}_{\text {peak }}^{>}(\mathbf{k}, \omega)$ behaves like $T / \mid \omega-$ $E_{\mathbf{k}} \mid$. At energies further away from the peak center, it decreases like $e^{-\left|\omega-E_{\mathbf{k}}\right| / T}$. Thus the width of the peak is of the order of the temperature and therefore corresponds to incoherent excitations. The vanishing of $\mathcal{A}(\mathbf{k}, \omega)$ for $\left|\omega-E_{\mathbf{k}}\right|<m$ is clearly unphysical (note that it cannot be seen in the figures, since $m$ is exponentially small). It would be suppressed by any finite life time in the bosonic propagator $\mathcal{D}$. The finite-temperature DOS suffers from the same artifact (i.e. $\rho(\omega)=0$ for $\left|\omega-\Delta_{0}\right|<m$ ).

The spectral weight of the peak at $E_{\mathbf{k}}$ is

$$
\begin{aligned}
\int d \omega \mathcal{A}_{\text {peak }}^{>}(\mathbf{k}, \omega) & =u_{\mathbf{k}}^{2} \frac{g}{2 \pi c} T \ln \left(\frac{T}{m}\right) \\
& =u_{\mathbf{k}}^{2}\left(1-\frac{g}{g_{c}}\right) .
\end{aligned}
$$

where the last result is obtained using Eq. (51). The spectral weight of the peak turns out to be temperature independent and equal to $u_{\mathbf{k}}^{2} n_{0}\left(n_{0}=1-g / g_{c}\right)$, which is nothing else but the Bogoliubov QP weight in 


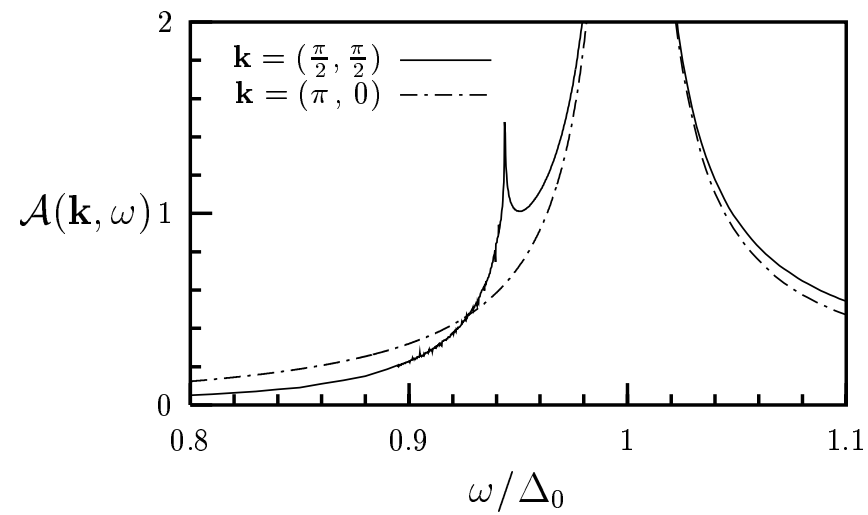

FIG. 8: Finite-temperature spectral function at weak coupling for two different points of the non-interacting Fermi surface. For $\mathbf{k}$ close to $(\pi / 2, \pi / 2)$ a second peak appears below $\Delta_{0}$ (see text).

the ground state. We conclude that the peak is an incoherent precursor of the zero-temperature Bogoliubov QP peak. As the temperature decreases, it retains its spectral weight but becomes sharper and sharper, and eventually becomes a Dirac peak at $T=0$. As expected, the spectral function evolves continuously when $T \rightarrow 0$. As in the zero-temperature case, the dependence of $n_{0}$ upon $U$ describes the transfer of spectral weight from the Bogoliubov QP's to the incoherent excitation background when the Coulomb repulsion increases.

The approximation (73) suggests that the peak in $\mathcal{A}(\mathbf{k}, \omega)$ should exhibit the same features, regardless of the location of $\mathbf{k}$ on the non-interacting Fermi surface. Numerical calculations confirm this conclusion, with one exception. For wave-vectors near $(\pi / 2, \pi / 2)$, a second (smaller) peak appears at low energy (Fig. 8). From a mathematical point of view, it is due to the vanishing of the first-order derivative of the argument of the delta function in Eq. (72), which occurs for $\nabla_{\mathbf{q}} \omega_{\mathbf{q}}=\nabla_{\mathbf{q}} E_{\mathbf{k}-\mathbf{q}}$. The energy at which the integration contour in the $\mathbf{q}$ plane, defined by the delta function, passes through this point can be estimated to be $\Delta_{0} \sqrt{1-\left(c /\left|\mathbf{v}_{\mathbf{k}}\right|\right)^{2}}$, where $\mathbf{v}_{\mathbf{k}}=\nabla_{\mathbf{k}} \epsilon_{\mathbf{k}}$ is the free-fermion velocity. For wave-vectors verifying $\left|\mathbf{v}_{\mathbf{k}}\right|<c$, i.e. sufficiently close to the Van-Hove singularities, the second peak disappears. We believe this second peak to be an artifact of our lowest-order approximation in the pseudo-fermion-boson interaction.

\section{Pseudogap vs Mott-Hubbard gap}

As shown in Figs. 4.5, the spectral function $\mathcal{A}(\mathbf{k}, \omega)$ extends below the HF energy $E_{\mathbf{k}}$ (and above $-E_{\mathbf{k}}$ for $\omega<$ $0)$ at finite temperature. The corresponding contribution to $\mathcal{A}(\mathbf{k}, \omega)$ is given by [see Eq. (61)]

$$
\int_{\mathbf{q}} \frac{g c}{2 \omega_{\mathbf{q}}} n_{B}\left(\omega_{\mathbf{q}}\right) \quad\left[u_{\mathbf{k}-\mathbf{q}}^{2} \delta\left(\omega+\omega_{\mathbf{q}}-E_{\mathbf{k}-\mathbf{q}}\right)\right.
$$

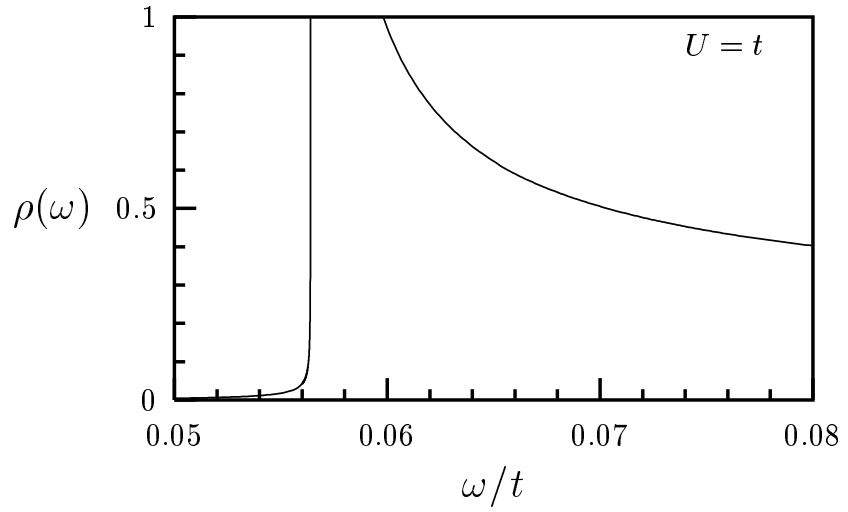

FIG. 9: Finite-temperature $\operatorname{DOS} \rho(\omega)$ at weak coupling: $U=$ $t, T=\Delta_{0} / 5$ (pseudogap phase). At $\omega=0$ the DOS is finite but exponentially small [Eq. (76)].

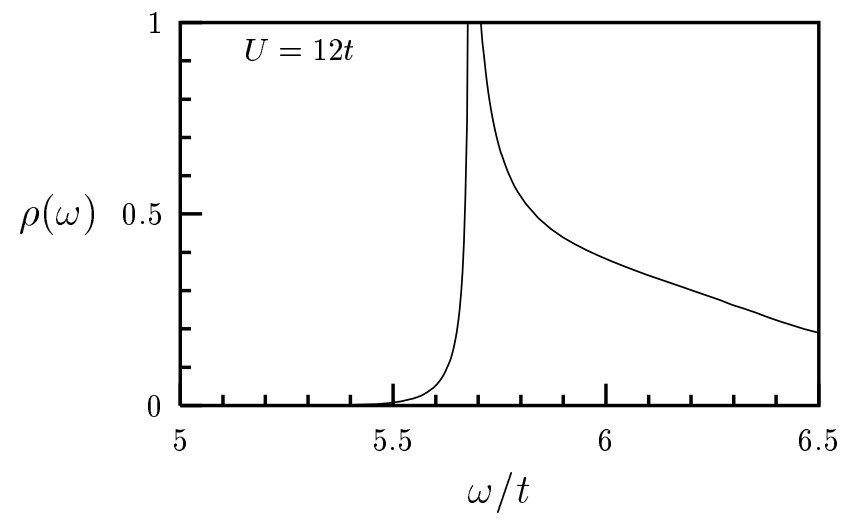

FIG. 10: Finite-temperature DOS $\rho(\omega)$ at strong coupling: $U=12 t, T=J / 5$ (Mott-Hubbard insulator).

$$
\left.+v_{\mathbf{k}-\mathbf{q}}^{2} \delta\left(\omega-\omega_{\mathbf{q}}+E_{\mathbf{k}-\mathbf{q}}\right)\right] .
$$

The presence of the Bose occupation number $n_{B}\left(\omega_{\mathbf{q}}\right)$ shows that the low-energy fermion states $\left(|\omega|<E_{\mathbf{k}}\right)$ are due to thermal bosons, i.e. thermally excited spin fluctuations. A fermion added to the system with momentum $\mathbf{k}$ and energy $|\omega|<E_{\mathbf{k}}$ can propagate by absorbing a thermal boson of energy $\omega_{\mathbf{q}}$ and emitting a pseudo-fermion with energy $E_{\mathbf{k}-\mathbf{q}}=\omega+\omega_{\mathbf{q}}$.

The lowest fermion energies are obtained by solving $\omega=E_{\mathbf{k}-\mathbf{q}}-\omega_{\mathbf{q}}$ (or $\left.\omega=-E_{\mathbf{k}+\mathbf{q}}+\omega_{\mathbf{q}}\right)$. In the weak coupling limit, $\max _{\mathbf{q}}\left(\omega_{\mathbf{q}}\right)=c \Lambda \sim 2 \Delta_{0}$ and $E_{\mathbf{k}-\mathbf{q}} \sim E_{\mathbf{k}}$. Thus there is spectral weight at zero energy: the spectral function and the density of states exhibit a pseudogap (Figs.4and 9). Note that the DOS remains exponentially small at low energy:

$$
\rho(\omega) \sim e^{\frac{-\Delta_{0}}{T}} \cosh \left(\frac{\omega}{T}\right),|\omega| \ll \Delta_{0} .
$$

This result differs from pseudogap theories based on Gaussian spin fluctuations which find a much weaker suppression of the density of states at low energy. ${ }^{48} \mathrm{It}$ bears some similarities with the results obtained by Bar- 


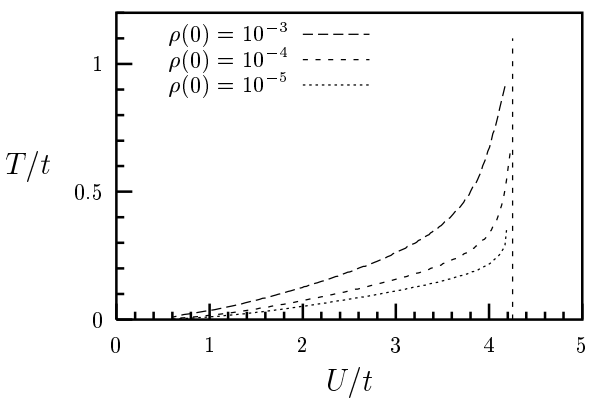

FIG. 11: Lines $\rho(\omega=0)=$ const in the $(U, T)$ plane. The vertical line corresponds to $\rho(\omega=0)=0$.

tosch and Kopietz for fermions coupled to classical phase fluctuations in incommensurate Peierls chains. ${ }^{51}$ In the strong coupling limit, thermally excited spin fluctuations lead to a small reduction of the zero-temperature gap since $c \Lambda \sim J \ll E_{\mathbf{k}} \sim U / 2$. The system is a MottHubbard insulator with a gap $2 \Delta_{0}$ of order $U$ (Figs. 5 and 10i).

A last comment is in order here. Since the system is in the renormalized classical regime, it is tempting to treat the NL $\sigma \mathrm{M}$ in the classical limit (which amounts to neglecting the quantum (temporal) fluctuations of the Néel field $\mathbf{n}$ ). Such an approach is expected to be at least qualitatively correct for the low-energy bosons $\left(\omega_{\mathbf{q}} \lesssim T\right)$ and should then give a good approximation of $\mathcal{A}(\mathbf{k}, \omega)$ in the vicinity of the peaks around $\omega= \pm E_{\mathbf{k}}$. Retaining only the $\omega_{\nu}=0$ contribution in Eq. (59), one finds

$$
\begin{array}{r}
\mathcal{A}_{\mathrm{cl}}(\mathbf{k}, \omega)=T \int_{\mathbf{q}} \frac{g c}{\omega_{\mathbf{q}}^{2}}\left[u_{\mathbf{k}-\mathbf{q}}^{2} \delta\left(\omega-E_{\mathbf{k}-\mathbf{q}}\right)\right. \\
\left.+v_{\mathbf{k}-\mathbf{q}}^{2} \delta\left(\omega+E_{\mathbf{k}-\mathbf{q}}\right)\right] .
\end{array}
$$

Eq. (77) can also be obtained from Eq. (61) by using $n_{B}\left(\omega_{\mathbf{q}}\right)+1 \sim n_{B}\left(\omega_{\mathbf{q}}\right) \sim T / \omega_{\mathbf{q}} \gg 1$ and neglecting the term $\pm \omega_{\mathbf{q}}$ in the argument of the delta functions. It is readily seen that the classical calculation does not reproduce the pseudogap, since $\mathcal{A}_{\mathrm{cl}}(\mathbf{k}, \omega)$ vanishes for $|\omega|<E_{\mathbf{k}}$. Although the pseudogap originates from thermally excited spin fluctuations in the renormalized classical regime, a fully quantum-mechanical calculation of $\mathcal{A}(\mathbf{k}, \omega)$ turns out to be necessary to account for the presence of low-energy fermion excitations.

\section{Finite-temperature metal-insulator transition}

We conclude from the results of Sec. IVB that our approach predicts a finite-temperature metal-insulator transition between a pseudogap phase and a MottHubbard insulator as the strength of the Coulomb interaction increases: at a critical value $U_{c}$, the density of states at zero energy $\rho(\omega=0)$ vanishes and the pseudogap becomes a Mott-Hubbard gap (Fig. 3). $U_{c}$ is obtained by equating the minimum energy $\Delta_{0}$ of a $\mathrm{HF}$ fermion to the maximum energy of a Schwinger boson $\sqrt{m^{2}+c^{2} \Lambda^{2}}$. For $T \rightarrow 0$ the result is $U_{c} \simeq 4.25 t$. It should be noted that the NL $\sigma \mathrm{M}$, which is a low-energy theory, does not allow us to describe accurately the highenergy Schwinger bosons (with $|\mathbf{q}| \sim \Lambda$ ) and in turn the low-energy fermion excitations. In particular, the critical value of $U$ calculated above depends on the cut-off procedure used in the NL $\sigma \mathrm{M}$. Note also that we do not know at which temperature and how the metal-insulator transition ends.

Fig. 11] shows the lines $\rho(\omega=0)=$ const in the $(U, T)$ plane. Our results are in (semi-quantitative) agreement with the numerical calculation of Moukouri et al. ${ }^{64}$ Using the criterion $\rho(\omega=0)<10^{-2} /(2 t)$ to identify the Mottinsulating phase, these authors concluded that the system is always insulating at low (but finite) temperature even in the weak-coupling limit, which seems to invalidate the Slater scenario as the mechanism for the metalinsulator transition (which requires $T_{\mathrm{MIT}}=T_{\mathrm{N}}=0$ ). Our approach shows that the results of Ref. 64 are not in contradiction with a Slater scenario at weak coupling, but merely reflect the exponential suppression of the density of states due to the presence of a pseudogap. A similar conclusion was reached in Ref. 65 .

\section{ATTRACTIVE HUBBARD MODEL}

In this section, we show that the results obtained in the previous sections translate directly to the attractive Hubbard model. The latter is defined by the Hamiltonian

$$
H=-\sum_{\mathbf{r}, \sigma} c_{\mathbf{r} \sigma}^{\dagger}(\hat{t}+\mu) c_{\mathbf{r} \sigma}-U \sum_{\mathbf{r}} c_{\mathbf{r} \uparrow}^{\dagger} c_{\mathbf{r} \uparrow} c_{\mathbf{r} \downarrow}^{\dagger} c_{\mathbf{r} \downarrow},
$$

where $-U(U \geq 0)$ is the on-site attraction. $\mu=-U / 2$ at half-filling.

Under the particle-hole transformation 67

$$
c_{\mathbf{r} \downarrow} \rightarrow(-1)^{\mathbf{r}} c_{\mathbf{r} \downarrow}^{\dagger}, \quad c_{\mathbf{r} \downarrow}^{\dagger} \rightarrow(-1)^{\mathbf{r}} c_{\mathbf{r} \downarrow},
$$

the Hamiltonian becomes (up to a constant term)

$$
\begin{aligned}
H= & -\sum_{\mathbf{r}, \sigma} c_{\mathbf{r} \sigma}^{\dagger}(\hat{t}+U / 2) c_{\mathbf{r} \sigma}+U \sum_{\mathbf{r}} c_{\mathbf{r} \uparrow}^{\dagger} c_{\mathbf{r} \uparrow} c_{\mathbf{r} \downarrow}^{\dagger} c_{\mathbf{r} \downarrow} \\
& -(\mu+U / 2) \sum_{\mathbf{r}}\left(c_{\mathbf{r} \uparrow}^{\dagger} c_{\mathbf{r} \uparrow}-c_{\mathbf{r} \downarrow}^{\dagger} c_{\mathbf{r} \downarrow}\right),
\end{aligned}
$$

and the charge-density and pairing operators transform as

$$
\begin{aligned}
\rho_{\mathbf{r}} & =\sum_{\sigma} c_{\mathbf{r} \sigma}^{\dagger} c_{\mathbf{r} \sigma} \rightarrow 2 S_{\mathbf{r}}^{z}+1, \\
\Delta_{\mathbf{r}} & =c_{\mathbf{r} \downarrow} c_{\mathbf{r} \uparrow} \rightarrow(-1)^{\mathbf{r}} S_{\mathbf{r}}^{-}, \\
\Delta_{\mathbf{r}}^{\dagger} & =c_{\mathbf{r} \uparrow}^{\dagger} c_{\mathbf{r} \downarrow}^{\dagger} \rightarrow(-1)^{\mathbf{r}} S_{\mathbf{r}}^{+},
\end{aligned}
$$

where $\mathbf{S}_{\mathbf{r}}=c_{\mathbf{r}}^{\dagger} \boldsymbol{\sigma} c_{\mathbf{r}} / 2$ and $S_{\mathbf{r}}^{ \pm}=S_{\mathbf{r}}^{x} \pm i S_{\mathbf{r}}^{y}$. The transformed Hamiltonian (80) corresponds to the repulsive 


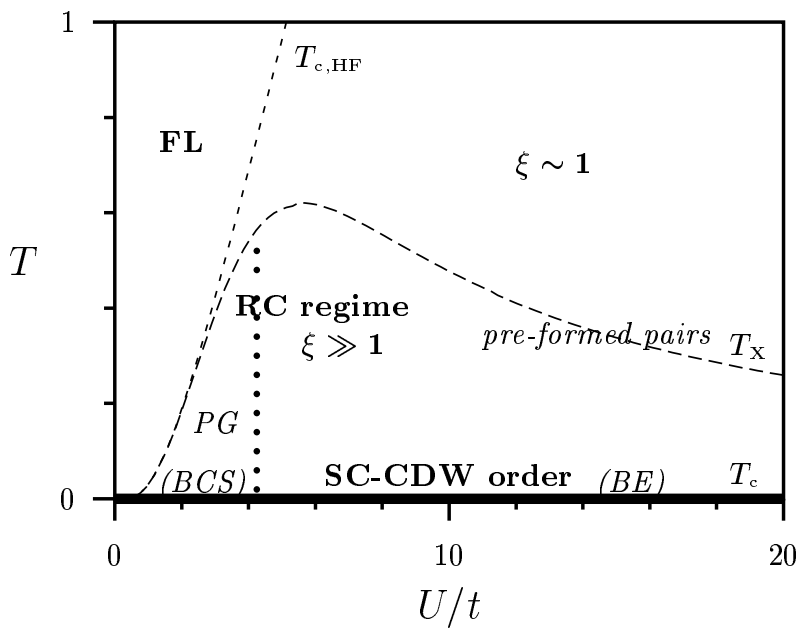

FIG. 12: Phase diagram of the 2D half-filled Hubbard model with an attractive interaction $-U(U \geq 0)$. $T \gtrsim T_{c}^{\mathrm{HF}}$ : Fermi liquid (FL) phase; $T_{\mathrm{X}} \lesssim T \lesssim T_{c}^{\mathrm{HF}}$ : preformed pairs with no superfluid or charge-density-wave order $(\xi \sim 1) ; T \lesssim T_{\mathrm{X}}$ : renormalized classical $(\mathrm{RC})$ regime $(\xi \gg 1) ; T=0$ : superconducting (SC) and charge-density-wave (CDW) long-range orders $(U \ll 4 t$ : BCS limit, $U \gg 4 t$ : Bose-Einstein (BE) limit). The dotted line is obtained from the vanishing of the tunneling DOS $\rho(\omega=0)$ at zero energy. All lines, except $T_{c}=0$ (thick solid line), are crossover lines.

half-filled Hubbard model with a uniform magnetic field $\mu+U / 2$ along the $z$ axis coupled to the fermion spins. At half-filling $(\mu=-U / 2)$, the latter vanishes and the Hamiltonian (80) reduces to the one studied in the previous sections. Thus, in the attractive model, $\mathbf{q}=\boldsymbol{\pi}$ charge and $\mathbf{q}=0$ pairing fluctuations combine to form an order parameter with $\mathrm{SO}(3)$ symmetry. Away from half-filling, the degeneracy between charge and pairing fluctuations is lifted (by the uniform magnetic field $\mu+U / 2$ in the repulsive model), and the (superconducting) order parameter exhibits $\mathrm{SO}(2)$ symmetry at low temperature. As a result, there is a Berezinskii-Kosterlitz-Thouless phase transition to a superconducting state at a finite temperature $T_{\mathrm{BKT}}, \frac{75,76.77}{7}$

In the following, we consider only the half-filled case where the attractive model maps onto the repulsive model studied in the present work. Since the Green's function and the spectral function are invariant under the particle-hole transformation (79), we can directly apply the results obtained in the previous sections. The phase diagram is shown in Fig. 12 The crossover lines are the same as in Fig. 3, but their physical meaning is different. Below the $\mathrm{HF}$ transition temperature $T_{c}^{\mathrm{HF}}$, the $\mathrm{SO}(3)$ order parameter $\left(\rho_{\mathbf{q}=\boldsymbol{\pi}}, \Delta_{\mathbf{q}=0}\right)$ acquires a finite amplitude $\Delta_{0}$. This corresponds to the appearance of bound particle-hole and particle-particle pairs with a size $\xi_{0} \sim t / \Delta_{0}$. Below $T_{X}$, directional correlations of the order parameter $\left(\rho_{\mathbf{q}=\boldsymbol{\pi}}, \Delta_{\mathbf{q}=0}\right)$ start to grow exponentially (renormalized classical regime) and eventually longrange order sets in at the $T_{c}=0$ phase transition. Be- cause of the $\mathrm{SO}(3)$ symmetry, the ground state can have any combination of superconducting and charge-densitywave long-range orders. As $U$ increases, the ground state smoothly evolve from the BCS to the Bose-Einstein limits. In the weak-coupling limit $(U \ll 4 t)$, there is a pseudogap regime at finite temperature due to the directional fluctuations of the $\mathrm{SO}(3)$ order parameter. In the strongcoupling limit $(U \gg 4 t)$, between $T_{c}^{\mathrm{HF}}$ and $T_{X}$, there is a regime of preformed (local) particle-particle pairs with no superfluid or charge-density-wave short-range order $(\xi \sim 1)$. Only below $T_{X}$ do these bosonic pairs begin to develop short-range order. At $T=0$, the particleparticle pairs Bose condense and/or localize, thus giving rise to superfluid and/or charge-density-wave long-range orders. The dotted line in Fig. 12 is obtained from the vanishing of the tunneling DOS $\rho(\omega=0)$ at zero energy.

\section{SUMMARY AND CONCLUSION}

We have presented an approach to the 2D half-filled Hubbard model which describes both collective spin fluctuations and single-particle properties for any value of the Coulomb repulsion $U$. It is valid below a crossover temperature $T_{X}$ where amplitude fluctuations of the $\mathrm{AF}$ order parameter are frozen out and $\mathrm{AF}$ short-range order starts to grow exponentially (renormalized classical regime).

The magnetic phase diagram is obtained from a $\mathrm{NL} \sigma \mathrm{M}$ that is derived from the Hubbard model. The parameters of the NL $\sigma \mathrm{M}$, the bare spin stiffness $\rho_{s}^{0}$ and the spin-wave velocity $c$, are expressed in terms of the mean-value of the kinetic energy and current-current correlation functions in the HF state. The model is solved by a saddle-point approximation within the $\mathrm{CP}^{1}$ representation where the Néel field is represented by two Schwinger bosons. BoseEinstein condensation of the Schwinger bosons at zero temperature signals the appearance of AF long-range order. At finite temperature (below $T_{X}$ ), the system is in a renormalized classical regime where the AF correlation length $\xi$ is exponentially large. The single-particle properties are obtained by writing the fermion field in terms of a Schwinger boson and a pseudo-fermion whose spin is quantized along the (fluctuating) Néel field. This decomposition allows us to approximate the fermion Green's function by the product (in real space) of the Schwinger boson propagator (which is obtained from the $\mathrm{NL} \sigma \mathrm{M}$ ) and the HF fermionic propagator.

Our results are summarized in Fig. 3, which shows the phase diagram of the 2D half-filled Hubbard model, and Figs. 4 10. At weak coupling and zero temperature, our theory clearly describes a Slater antiferromagnet with an exponentially small AF gap, well-defined Bogoliubov QP's carrying most of the spectral weight, and an incoherent excitation background at higher energy. As $U$ increases, the Slater antiferromagnet progressively evolves into a Mott-Heisenberg antiferromagnet with an AF gap of order $U$ and a significant fraction of spectral weight 
transferred from the Bogoliubov QP's to the incoherent excitation background. At finite temperature, the Bogoliubov QP's disappear and only incoherent excitations survive. Nevertheless, precursors of the zero-temperature Bogoliubov QP's show up as sharp peaks in the fermion spectral function, with a width of order $T$. The presence of thermal spin fluctuations gives rise to fermionic states below the zero-temperature AF gap. At weak coupling, the latter is completely filled and replaced by a pseudogap. The DOS $\rho(\omega)$ remains however exponentially small at low energy. At strong coupling and finite temperature $\left(0<T \lesssim T_{X} \sim J\right)$, the system is a paramagnetic Mott-Hubbard insulator in a renormalized classical regime of spin fluctuations. At higher temperature, $T_{X} \sim J \lesssim T \lesssim T_{\mathrm{N}}^{\mathrm{HF}}$, the system is characterized by the presence of preformed local moments without AF short-range order. Thus our theory predicts a metal-insulator transition at finite temperature between a pseudogap phase at weak coupling and a Mott-Hubbard insulator at strong coupling. For the 3D Hubbard model, we expect a similar phase diagram, but with $T_{X}$ replaced by a true transition line $T_{c}$ between a paramagnetic phase and an AF phase. The weak coupling pseudogap phase therefore appears as a consequence of the low dimensionality of the system and the high symmetry (i.e. $\mathrm{SO}(3)$ ) of the AF order parameter.

At half-filling the attractive and repulsive Hubbard models can be mapped onto one another by a canonical transformation so that our results also apply to the attractive case. AF fluctuations in the repulsive model correspond to $\mathbf{q}=\boldsymbol{\pi}$ charge and $\mathbf{q}=0$ pairing fluctuations in the attractive model. The corresponding phase diagram is discussed in Sec. D (see Fig. 12).

Besides its validity both at weak and strong coupling, our approach differs from previous weak-coupling theories ${ }^{33.34 .35 .36 .37 .38 .39 .40 .41 .42 .43 .44 .45 .46 .47}$ of the pseudogap phase in two respects. First, it takes spin fluctuations into account within a highly non-Gaussian theory (the $\mathrm{NL} \sigma \mathrm{M})$ and is valid at low temperature $(0 \leq T \ll$ $\left.T_{X}\right)$. On the contrary, most of the other approaches assume Gaussian spin fluctuations so that their range of validity is restricted to $T \sim T_{X}$. Second, our NL $\sigma \mathrm{M}$ approach is an expansion about the AF ordered state which is a valid starting point in presence of AF short-range order. When calculating fermion propagators, we have to consider HF pseudo-fermions interacting with Schwinger bosons whose dynamics is determined by the NL $\sigma \mathrm{M}$. Since the HF pseudo-fermions are gapped, we expect a perturbative expansion in the pseudo-fermion-boson interaction to be well-behaved. Our results were obtained to lowest order where the fermion Green's function is given by the product (in real space) of the HF fermionic propagator and the Schwinger boson propagator (which is obtained from the NL $\sigma \mathrm{M}$ ). This should be contrasted with perturbative treatments applied to free fermions interacting with soft collective fluctuations where no small expansion parameter is available.

Our NL $\sigma \mathrm{M}$ approach is reminiscent of slave-fermion theories ${ }^{60.61 .62}$ where the fermion is written as the product of a spinless pseudo-fermion and a Schwinger boson carrying the spin degrees of freedom. Slave-fermion theories apply to the $t-J$ model where the Hilbert space is truncated by forbidding double occupancy of the lattice sites. In our work, the pseudo-fermion also carries a spin, which is a necessary condition to describe both the weak and strong coupling regimes.

Our approach bears also some analogies with the work of Gusynin et al ${ }^{52.78 .79}$ on 2D fermion systems with an attractive interaction. These authors use a "modulusphase" representation for the $\mathrm{SO}(2)$ superconducting order parameter which is analog to our "amplitudedirection" representation of the $\mathrm{SO}(3) \mathrm{AF}$ order parameter. At low temperature, the phase of the superconducting order parameter is governed by a $\mathrm{SO}(2)$ sigma model. The fermion Green's function is calculated both above and below the Berezinskii-KosterlitzThouless phase transition $T_{\mathrm{BKT}}$ by writing the fermion field as the product of a pseudo-fermion and a bosonic field which is related to the phase of the order parameter. As in our work, a simple decoupling procedure between pseudo-fermions and bosons is used. A pseudogap phase is found both above and below $T_{\mathrm{BKT}}$. Gusynin et al. also point out the necessity to perform a fully quantum-mechanical calculation to describe the pseudogap phase ${ }^{79}$ The main difference with our work comes from the $\mathrm{SO}(2)$ symmetry of the order parameter which leads to a finite-temperature BerezinskiiKosterlitz-Thouless phase transition.

Let us now mention some limitations of our approach. (i) The feedback of spin fluctuations on (pseudo)fermions is not fully taken into account. As a result, we miss important effects, like the renormalization of the zero-temperature HF gap $\Delta_{0}$ by quantum spin fluctuations. (ii) The crossover temperature $T_{X}$, which is identified to the $\mathrm{HF}$ transition temperature $T_{\mathrm{N}}^{\mathrm{HF}}$ at weak coupling, is overestimated. Due to Kanamori screening effects, $T_{X}$ should be smaller than $T_{\mathrm{N}}^{\mathrm{HF}}, 13.65$ (iii) The NL $\sigma \mathrm{M}$ approach is restricted to low temperature $\left(T \ll T_{X}\right)$. In particular, it does not give access to the crossover regime between the Fermi liquid and the pseudogap phase at weak coupling. This regime is characterized, as the temperature decreases, by the suppression of Landau's QP's. (iv) At finite temperature, we predict a metal-insulator transition between a pseudogap phase and a Mott-Hubbard insulator. However, being a low-energy theory, the NL $\sigma \mathrm{M}$ does not allow to study the finite-temperature metal-insulator transition in detail (see Sec. IV).

But the main shortcoming of our approach is that it does not distinguish between Bogoliubov and MottHubbard bands. We find a single energy scale $\left(\Delta_{0}\right)$ in the density of states $\rho(\omega)$ and the spectral function $\mathcal{A}(\mathbf{k}, \omega)$. On physical grounds, we expect instead two energy scales, namely $\Delta_{0}$ and $U / 2$, corresponding to Bogoliubov bands (or precursors thereof at finite temperature) and Mott-Hubbard bands, respectively ${ }^{13}$ In 
the weak coupling limit, $\Delta_{0}$ depends crucially on the nesting properties of the Fermi surface (Slater antiferromagnetism). On the other hand, the energy scale $U / 2$ has a purely local origin, which is independent of the Fermi surface geometry, and is associated with the MottHubbard localization. A proper description of the MottHubbard localization would require to treat the charge fluctuations beyond the HF approximation for the $\Delta_{c}$ field (Sec. III). In the strong-coupling limit, charge fluctuations are frozen out. This is the reason why the HF saddle point for the amplitude fields $\Delta_{c}$ and $\Delta_{s}$ provides an accurate description of the local moments (whose direction is given by the $\boldsymbol{\Omega}_{\mathbf{r}}$ field) which form in the strong coupling limit ${ }^{80}$ Note that for $U \gg 4 t, \Delta_{0} \rightarrow U / 2$ so that the system is characterized by a single energy scale. At intermediate coupling $(U \sim 8 t)$, a four-peak structure corresponding to the simultaneous presence of Bogoliubov and Mott-Hubbard bands has been observed in numerical simulations ${ }^{81.82}$ and analytical studies ${ }^{13.83}$ of the Hubbard model. Although it misses some aspects of the Mott-Hubbard localization, in particular at intermediate coupling, we believe that our theory captures the main features of the physics of the $2 \mathrm{D}$ half-filled Hubbard model.

There are several directions in which this work could be further developed. The most obvious one is to consider situations where antiferromagnetism is frustrated due to either a non-bipartite lattice or a finite next-neighbor hopping amplitude. Doping would also induce magnetic frustration. This opens up the possibility to stabilize more exotic magnetic orders (e.g. a non-collinear order), and/or to reach the quantum disordered and quantum critical regimes of the NL $\sigma \mathrm{M}$ (Fig. 2) and study the cor- responding fermion spectral functions.

\section{Acknowledgments}

We would like to thank A.M.-S. Tremblay for numerous discussions and for pointing out Ref. 52 .

\section{APPENDIX A: HF CURRENT-CURRENT CORRELATION FUNCTION}

In this appendix we calculate the static uniform current-current correlation function

$$
\Pi_{\mu \mu^{\prime}}^{\nu \nu^{\prime}}=\left\langle j_{\mu}^{\nu}(\mathbf{0}, 0) j_{\mu^{\prime}}^{\nu^{\prime}}(\mathbf{0}, 0)\right\rangle_{\mathrm{HF}} .
$$

From the definition of the current $j_{\mu}^{\nu}$ [Eqs. (25:26)], we see that its zero-frequency zero-momentum Fourier transform involved in Eq. A1 is given by

$$
j_{\mu}^{\nu}(\mathbf{0}, 0)=\frac{1}{\sqrt{\beta \mathcal{N}}} \sum_{\mathbf{k}, \omega} v_{\mu}(\mathbf{k}) \Phi_{\mathbf{k} \omega}^{\dagger} \sigma_{\nu} \Phi_{\mathbf{k} \omega}
$$

where

$$
\begin{aligned}
& v_{0}(\mathbf{k})=1 \\
& v_{\mu}(\mathbf{k})=2 t \sin \left(k_{\mu}\right), \quad \mu=x, y .
\end{aligned}
$$

Using the Wick's theorem to evaluate HF averages of $\Phi$ fields, we can express $\Pi_{\mu \mu^{\prime}}^{\nu \nu^{\prime}}$ as

$$
\Pi_{\mu \mu^{\prime}}^{\nu \nu^{\prime}}=-\frac{1}{\beta \mathcal{N}} \sum_{\substack{\mathbf{k}, \omega \\ \mathbf{k}^{\prime}, \omega^{\prime}}} v_{\mu}(\mathbf{k}) v_{\mu^{\prime}}\left(\mathbf{k}^{\prime}\right) \operatorname{Tr}\left[\sigma_{\nu}\left\langle\Phi_{\mathbf{k} \omega} \Phi_{\mathbf{k}^{\prime} \omega^{\prime}}^{\dagger}\right\rangle \sigma_{\nu^{\prime}}\left\langle\Phi_{\mathbf{k}^{\prime} \omega^{\prime}} \Phi_{\mathbf{k} \omega}^{\dagger}\right\rangle\right]
$$

where $\operatorname{Tr}$ denotes the trace with respect to the spin indices. Writing the HF propagator [Eq. (11)] as

$$
\begin{aligned}
-\left\langle\Phi_{\mathbf{k} \omega} \Phi_{\mathbf{k}^{\prime} \omega^{\prime}}^{\dagger}\right\rangle & =\delta_{\omega, \omega^{\prime}}\left[\delta_{\mathbf{k}, \mathbf{k}^{\prime}} G(\mathbf{k}, \omega)+\delta_{\mathbf{k}, \mathbf{k}^{\prime}+\boldsymbol{\pi}} \sigma_{3} F(\mathbf{k}, \omega)\right], \\
G(\mathbf{k}, \omega) & =\frac{-i \omega-\epsilon_{\mathbf{k}}}{\omega^{2}+E_{\mathbf{k}}^{2}}, \quad F(\mathbf{k}, \omega)=\frac{\Delta_{0}}{\omega^{2}+E_{\mathbf{k}}^{2}},
\end{aligned}
$$

and using $\operatorname{Tr}\left(\sigma_{\nu} \sigma_{\nu^{\prime}}\right)=2 \delta_{\nu, \nu^{\prime}}, \operatorname{Tr}\left(\sigma_{3} \sigma_{\nu} \sigma_{3} \sigma_{\nu^{\prime}}\right)=2 \delta_{\nu, \nu^{\prime}}\left(2 \delta_{\nu, 3}-1\right)$ and $F(\mathbf{k}+\boldsymbol{\pi}, \omega)=F(\mathbf{k}, \omega)$, we obtain

$$
\Pi_{\mu \mu^{\prime}}^{\nu \nu^{\prime}}=-\frac{2 \delta_{\nu, \nu^{\prime}}}{\beta \mathcal{N}} \sum_{\mathbf{k}, \mathbf{k}^{\prime}, \omega} v_{\mu}(\mathbf{k}) v_{\mu^{\prime}}\left(\mathbf{k}^{\prime}\right)\left[\delta_{\mathbf{k}, \mathbf{k}^{\prime}} G(\mathbf{k}, \omega)^{2}+\delta_{\mathbf{k}, \mathbf{k}^{\prime}+\boldsymbol{\pi}}\left(2 \delta_{\nu, 3}-1\right) F(\mathbf{k}, \omega)^{2}\right] .
$$

$\Pi_{\mu \mu^{\prime}}^{\nu \nu^{\prime}}$ is thus diagonal in $\nu$ and $\nu^{\prime}$. One can show that it is also diagonal in $\mu$ and $\mu^{\prime}$. Indeed, whenever these two indices are different, the rhs of (A8) is odd in $k_{x}$ or $k_{y}$ and vanishes after wave-vector summation. Furthermore, $v_{0}(\mathbf{k}+\boldsymbol{\pi})=v_{0}(\mathbf{k})$ and $v_{\mu}(\mathbf{k}+\boldsymbol{\pi})=-v_{\mu}(\mathbf{k})$ for $\mu=x, y$, so that

$$
\Pi_{\mu \mu^{\prime}}^{\nu \nu^{\prime}}=-\frac{2 \delta_{\nu, \nu^{\prime}} \delta_{\mu, \mu^{\prime}}}{\beta \mathcal{N}} \sum_{\mathbf{k}, \omega} v_{\mu}(\mathbf{k})^{2}\left[G(\mathbf{k}, \omega)^{2}+\left(2 \delta_{\mu, 0}-1\right)\left(2 \delta_{\nu, 3}-1\right) F(\mathbf{k}, \omega)^{2}\right] \text {. }
$$

For $T \ll T_{\mathrm{N}}^{\mathrm{HF}}$, one can perform the Matsubara frequency summation in the zero-temperature limit. This gives

$$
=\frac{\Delta_{0}^{2}}{4 E_{\mathbf{k}}^{3}}
$$

$$
-\frac{1}{\beta} \sum_{\omega} G(\mathbf{k}, \omega)^{2}=\frac{1}{\beta} \sum_{\omega} F(\mathbf{k}, \omega)^{2}
$$


The only non-vanishing correlator functions are therefore

$$
\begin{aligned}
\Pi_{00}^{11} & =\Pi_{00}^{22} \\
& =\int_{\mathbf{k}} \frac{\Delta_{0}^{2}}{E_{\mathbf{k}}^{3}}, \\
\Pi_{x x}^{33} & =\Pi_{y y}^{33} \\
& =4 \Delta_{0}^{2} t^{2} \int_{\mathbf{k}} \frac{\sin ^{2} k_{x}}{E_{\mathbf{k}}^{3}} .
\end{aligned}
$$

\section{APPENDIX B: SU(2) GAUGE FIELD}

In this appendix we give a proof of Eqs. (35.36), relating the Néel and canting fields $\mathbf{n}_{\mathbf{r}}$ and $\mathbf{L}_{\mathbf{r}}$ to the gauge field $A_{\mu \mathbf{r}}^{\nu}$ and the rotated canting field $\mathbf{l}_{\mathbf{r}}$. Let us recall the definition of the gauge field:

$$
A_{\mu \mathbf{r}}=i R_{\mathbf{r}}^{\dagger} \partial_{\mu} R_{\mathbf{r}}, \quad \mu=t, x, y .
$$

The index $t$ stands for real-time derivation. Imaginarytime results are obtained using $\tau=i t$. The $\mathrm{SU}(2) / \mathrm{U}(1)$ rotation matrix $R_{\mathbf{r}}$ is defined, up to a U(1) gauge transformation $R_{\mathbf{r}} \rightarrow R_{\mathbf{r}} e^{i \alpha_{\mathbf{r}} \sigma_{3}}$, by

$$
\boldsymbol{\sigma} \cdot \mathbf{n}_{\mathbf{r}}=R_{\mathbf{r}} \sigma_{3} R_{\mathbf{r}}^{\dagger}
$$

which means that the $\mathrm{SO}(3)$ element $\mathcal{R}_{\mathbf{r}}$ associated to $R_{\mathbf{r}}$ maps $\mathbf{u}_{z}$ onto $\mathbf{n}_{\mathbf{r}}$. The gauge field $A_{\mu \mathbf{r}}^{\nu}$ is a zero-trace Hermitian matrix which can be decomposed on Pauli matrices $\sigma_{\nu}$ :

$$
\begin{aligned}
A_{\mu \mathbf{r}} & =\sum_{\nu=1,2,3} A_{\mu \mathbf{r}}^{\nu} \sigma_{\nu} \\
& =\mathbf{A}_{\mu \mathbf{r}} \cdot \boldsymbol{\sigma}
\end{aligned}
$$

where the bold notation denotes the three-component vector $\left(A_{\mu}^{1}, A_{\mu}^{2}, A_{\mu}^{3}\right)$.

The main result of this appendix is the following general form for the $\mathbf{A}_{\mu \mathbf{r}}$ field:

$$
\begin{aligned}
\mathbf{A}_{\mu \mathbf{r}} & =\mathcal{R}_{\mathbf{r}}^{-1}\left(\frac{1}{2} \mathbf{n}_{\mathbf{r}} \wedge \partial_{\mu} \mathbf{n}_{\mathbf{r}}+\kappa_{\mu \mathbf{r}} \mathbf{n}_{\mathbf{r}}\right) \\
& =\frac{1}{2} \mathbf{u}_{z} \wedge \mathcal{R}_{\mathbf{r}}^{-1}\left(\partial_{\mu} \mathbf{n}_{\mathbf{r}}\right)+\kappa_{\mu \mathbf{r}} \mathbf{u}_{z}
\end{aligned}
$$

$\kappa_{\mu \mathbf{r}}$ is some function of position and time, fixed by the choice of a gauge. Notice however, that it cannot be any function, since it appears in the expression of the gaugefield density tensor, which must be zero.

Eqs. (35.36) follow quite easily. First, we have

$$
\sum_{\nu=1,2} A_{\mu \mathbf{r}}^{\nu 2}=\frac{1}{4}\left[\left.\mathcal{R}_{\mathbf{r}}^{-1}\left(\mathbf{n}_{\mathbf{r}} \wedge \partial_{\mu} \mathbf{n}_{\mathbf{r}}\right)\right|^{2}\right.
$$

$$
\begin{aligned}
& =\frac{1}{4}\left(\mathbf{n}_{\mathbf{r}} \wedge \partial_{\mu} \mathbf{n}_{\mathbf{r}}\right)^{2} \\
& =\frac{1}{4}\left(\partial_{\mu} \mathbf{n}_{\mathbf{r}}\right)^{2} .
\end{aligned}
$$

Using $\partial_{t}=i \partial_{\tau}$ we obtain Eq. (35). Second, recalling that the rotated canting vector $\mathbf{l}_{\mathbf{r}}=\mathcal{R}_{\mathbf{r}}^{-1} \mathbf{L}_{\mathbf{r}}$ has no component along $\mathbf{u}_{z}$, we can write

$$
\begin{aligned}
\sum_{\nu=1,2} A_{\mu \mathbf{r}}^{\nu} l_{\mathbf{r}}^{\nu} & =\mathbf{A}_{\mu \mathbf{r}} \cdot \mathbf{l}_{\mathbf{r}} \\
& =\frac{1}{2} \mathcal{R}_{\mathbf{r}}^{-1}\left(\mathbf{n}_{\mathbf{r}} \wedge \partial_{\mu} \mathbf{n}_{\mathbf{r}}\right) \cdot \mathcal{R}_{\mathbf{r}}^{-1}\left(\mathbf{L}_{\mathbf{r}}\right) \\
& =\frac{1}{2}\left(\mathbf{n}_{\mathbf{r}} \wedge \partial_{\mu} \mathbf{n}_{\mathbf{r}}\right) \cdot \mathbf{L}_{\mathbf{r}}
\end{aligned}
$$

hence Eq. (36).

We now give a derivation of Eq. (B5). The first step is to differentiate Eq. (B2). Derivatives of the rotation matrix are calculated using Eq. (B1) and the identity $\partial_{\mu} R_{\mathbf{r}}^{\dagger}=-R_{\mathbf{r}}^{\dagger}\left(\partial_{\mu} R_{\mathbf{r}}\right) R_{\mathbf{r}}^{\dagger}$ which results from the unitarity of $R_{\mathrm{r}}$. We obtain

$$
\begin{aligned}
\boldsymbol{\sigma} \cdot \partial_{\mu} \mathbf{n}_{\mathbf{r}} & =-i R_{\mathbf{r}} A_{\mu \mathbf{r}} \sigma_{3} R_{\mathbf{r}}^{\dagger}+i R_{\mathbf{r}} \sigma_{3} A_{\mu \mathbf{r}} R_{\mathbf{r}}^{\dagger} \\
& =-i R_{\mathbf{r}} A_{\mu \mathbf{r}} R_{\mathbf{r}}^{\dagger} R_{\mathbf{r}} \sigma_{3} R_{\mathbf{r}}^{\dagger}+i R_{\mathbf{r}} \sigma_{3} R_{\mathbf{r}}^{\dagger} R_{\mathbf{r}} A_{\mu \mathbf{r}} R_{\mathbf{r}}^{\dagger}
\end{aligned}
$$

Let us define a new field

$$
\begin{aligned}
\tilde{A}_{\mu \mathbf{r}} & =R_{\mathbf{r}} A_{\mu \mathbf{r}} R_{\mathbf{r}}^{\dagger} \\
& =\tilde{\mathbf{A}}_{\mu \mathbf{r}} \cdot \boldsymbol{\sigma}
\end{aligned}
$$

Using $\tilde{A}_{\mu \mathbf{r}}$ and Eq. (B2) we can rewrite Eq. (B8) as

$$
\begin{aligned}
\boldsymbol{\sigma} \cdot \partial_{\mu} \mathbf{n}_{\mathbf{r}} & =-i\left[\tilde{A}_{\mu \mathbf{r}}, \boldsymbol{\sigma} \cdot \mathbf{n}_{\mathbf{r}}\right] \\
& =-i\left[\boldsymbol{\sigma} \cdot \tilde{\mathbf{A}}_{\mu \mathbf{r}}, \boldsymbol{\sigma} \cdot \mathbf{n}_{\mathbf{r}}\right] \\
& =2 \boldsymbol{\sigma} \cdot\left(\tilde{\mathbf{A}}_{\mu \mathbf{r}} \wedge \mathbf{n}_{\mathbf{r}}\right)
\end{aligned}
$$

We have used the identity

$$
[\boldsymbol{\sigma} \cdot \mathbf{u}, \boldsymbol{\sigma} \cdot \mathbf{v}]=2 i \boldsymbol{\sigma} \cdot(\mathbf{u} \wedge \mathbf{v})
$$

Identifying the coefficients of $\boldsymbol{\sigma}$ in Eq. (B10) and vectormultiplying by $\mathbf{n}_{\mathbf{r}}$ we arrive at

$$
\tilde{\mathbf{A}}_{\mu \mathbf{r}}=\frac{1}{2} \mathbf{n}_{\mathbf{r}} \wedge \partial_{\mu} \mathbf{n}_{\mathbf{r}}+\left(\mathbf{n}_{\mathbf{r}} \cdot \tilde{\mathbf{A}}_{\mu \mathbf{r}}\right) \mathbf{n}_{\mathbf{r}}
$$

To conclude, it is sufficient to define the last term in Eq. B12 as $\kappa_{\mu \mathbf{r}}$ and to remark that, owing to the definition of $\tilde{A}_{\mu \mathbf{r}}$, we have $\tilde{\mathbf{A}}_{\mu \mathbf{r}}=\mathcal{R}_{\mathbf{r}} \mathbf{A}_{\mu \mathbf{r}}$.

\footnotetext{
1 J. Hubbard, Proc. R. Soc. London, Ser. A 276, 238 (1963).
}

${ }^{2}$ M.C. Gutzwiller, Phys. Rev. Lett. 10, 159 (1963). 
3 J. Kanamori, Prog. Theor. Phys. 30, 275 (1963).

4 For a general introduction to the Hubbard model, see F. Gebhard, The Mott metal-insulator transition (SpringerVerlag, 1997).

5 See, for instance, M. Imada, A. Fujimori, and Y. Tokura, Rev. Mod. Phys. 70, 1039 (1998).

6 See, for instance, J. Voit, Rep. Prog. Phys. 57, 977 (1994).

7 For a review, see A. Georges, G. Kotliar, W. Krauth, and M.J. Rozenberg, Rev. Mod. Phys. 68, 13 (1996).

8 J.E. Hirsh, Phys. Rev. B 31, 4403 (1985).

9 S.R. White, D.J. Scalapino, R.L. Sugar, E.Y. Loh, J.E. Gubernatis, and R.T. Scalettar, Phys. Rev. B 40, 506 (1989).

10 J.C. Slater, Phys. Rev. 82, 538 (1951).

11 A. Auerbach, Interacting Electrons and Quantum Magnetism (Springer-Verlag, New York, 1994).

12 N.D. Mermin and H. Wagner, Phys. Rev. Lett. 17, 1133 (1966).

13 Y.M. Vilk and A.-M.S. Tremblay, J. Phys. I (France) 7, 1309 (1997).

14 T. Moriya, Spin Fluctuations in Itinerant Electron Magnetism (Springer-Verlag, 1985).

15 J.R. Schrieffer, X.G. Wen, and S.C. Zhang, Phys. Rev. B 39, 11663 (1989).

16 A. Singh and Z. Tesanović, Phys. Rev. B 41, 614 (1990).

17 A.V. Chubukov and D.M. Frenkel, Phys. Rev. B 46, 11884 (1992).

18 G. Vignale and M.R. Hedayati, Phys. Rev. B 42, 786 (1990).

19 W. Brenig and A.P. Kampf, EuroPhys. Lett. 24, 679 (1993).

20 J. Altmann, W. Brenig, A.P. Kampf, and E. MüllerHartmann, Phys. Rev. B 52, 7395 (1995).

21 E. Dagotto, F. Ortolani, and D.J. Scalapino, Phys. Rev. B 46, 3183 (1992).

22 P.W. Leng, Z. Liu, E. Manousakis, M.A. Novotny, and P.E. Oppenheimer, Phys. Rev. B 46, 11779 (1992).

${ }^{23}$ G.S. Feng and S.R. White, Phys. Rev. B 46, 8691 (1992).

${ }^{24}$ See E. Dagotto, Rev. Mod. Phys. 66, 763 (1994), and references therein.

25 T. Moriya and K. Ueda, Adv. Phys. 49, 555 (2000).

26 T. Moriya, Y. Takahashi, and K. Ueda, J. Phys. Soc. Japan 59, 2905 (1990).

27 N.E. Bickers and D.J. Scalapino, Ann. Phys. (N.Y.) 193, 206 (1989).

28 S. Chakravarty, B.I.Halperin, and D.R. Nelson, Phys. Rev. B 39, 2344 (1989).

29 A.V. Chubukov, S. Sachdev, and J. Ye, Phys. Rev. B 49, 11919 (1994).

${ }^{30}$ M. Langer, J. Schmalian, S. Grabowski and K.H. Bennemann, Phys. Rev. Lett. 75, 4508 (1995).

31 J.J Deisz, D.W. Hess and J.W. Serene, Phys. Rev. Lett. 76, 1312 (1996).

${ }^{32}$ Y.M. Vilk and A.-M.S. Tremblay, EuroPhys. Lett. 33, 159 (1996).

33 P.A. Lee, T.M. Rice, and P.W. Anderson, Phys. Rev. Lett. 31, 462 (1973).

34 M.V. Sadovskii, Sov. Phys. JETP 39, 845 (1974).

35 M.V. Sadovskii, Sov. Phys. JETP 50, 989 (1979).

36 O. Tchernyshyov, Phys. Rev. B 59, 1358 (1999).

37 L. Bartosch and P. Kopietz, Phys. Rev. B 60, 15488 (1999).

38 A.J. Millis and H. Monien, Phys. Rev. B 61, 12496 (2000).

39 J. Schmalian, D. Pines, and B. Stojković, Phys. Rev. Lett. 80, 3839 (1998).

40 J. Schmalian, D. Pines, and B. Stojković, Phys. Rev. B 60,
667 (1999).

41 A.P. Kampf and J.R. Schrieffer, Phys. Rev. B 41, 6399 (1989).

42 A.P. Kampf and J.R. Schrieffer, Phys. Rev. B 42, 7967 (1990).

43 P. Monthoux and D. Pines, Phys. Rev. B 47, 6069 (1993).

44 B.L. Altshuler, L.B. Ioffe and A.J. Millis, Phys. Rev. B 52, 5563 (1995).

45 Y.M. Vilk, Phys. Rev. B 55, 3870 (1997).

46 Ar. Abanov and A.V. Chubukov, Phys. Rev. Lett. 84, 5608 (2000).

47 A. Posazhennikova and P. Coleman, preprint cond-mat/0209014

48 The failure of Gaussian spin fluctuation theories at low temperature has recently been emphasized by H. Monien, preprint cond-mat/0110178

49 N. Dupuis, Phys. Rev. B 65, 245118 (2002).

${ }^{50}$ H. Monien, Phys. Rev. Lett. 87, 126402 (2001).

${ }^{51}$ L. Bartosch and P. Kopietz, Phys. Rev. B 62, R16223 (2000).

${ }^{52}$ For a review, see V.M. Loktev, R.M. Quick, and S.G. Sharapov, Phys. Rep. 349, 1 (2001).

53 G. Grüner, Density Waves in Solids (Addison Wesley, 1994).

54 T. Timusk and B. Statt, Rep. Prog. Phys. 62, 61 (1999).

55 J. L. Tallon and J. W. Loram, Physica C, 349, 53 (2001).

${ }^{56}$ K. Borejsza and N. Dupuis, EuroPhys. Lett. 63, 722 (2003).

57 H.J. Schulz in The Hubbard Model, edited by D. Baeriswyl (Plenum, New York, 1995).

58 In quasi-1D systems, NL $\sigma$ M's have been derived in the weak-coupling limit by K. Sengupta and N. Dupuis, Phys. Rev. B 61, 13493 (2000); Y. Tomio, N. Dupuis, Y. Suzumura, Phys. Rev. B 64, 125123 (2001).

${ }^{59}$ For a review, see for example, G. Kotliar in Correlated Electrons Systems, V.J. Emery Ed. (World Scientific, Singapore, 1993).

60 C. Jayaprakash, H.R. Krishnamurthy and S. Sarker, Phys. Rev. B 40, 2610 (1989).

61 D. Yoshioka, J. Phys. Soc. Japan 58, 1516 (1989).

62 A. Auerbach and D.E. Larson, Phys. Rev. B 43, 7800 (1991).

63 Our approach is closer to slave-fermion theories since the spin degrees of freedom are represented by Schwinger bosons.

64 S. Moukouri and M. Jarrell, Phys. Rev. Lett. 87, 167010 (2001).

65 B. Kyung, J.S. Landry, D. Poulin, and A.M.-S. Tremblay, Phys. Rev. Lett. 90, 099702 (2003).

66 See also P.W. Anderson, Adv. Phys. 46, 3 (1997).

67 R. Micnas, J. Ranninger, and S. Robaszkiewicz, Rev. Mod. Phys. 62, 113 (1990).

68 Z.Y. Weng, C.S Ting and T.K. Lee, Phys. Rev. B 43, 3790 (1991).

${ }^{69}$ F.D.M. Haldane, Phys. Lett. 93A, 464 (1983).

70 The second-order expansion of the cosine in Eq. (19) includes a term proportional to $\partial_{\mu} A_{\mu \mathbf{r}}^{\nu}$, but the latter turns out to give a contribution of order $\left(\partial \mathbf{n}_{\mathbf{r}}\right)^{3}$ to the action, upon an appropriate rearrangement of the sum over $\mathbf{r}$.

$712 \Delta_{0}$ corresponds to the threshold energy for particle-hole excitations in the HF state. Since spin-wave modes cannot exist above this energy, a natural choice for the $\mathrm{NL} \sigma \mathrm{M}$ cutoff is $\Lambda \sim \min \left(1,2 \Delta_{0} / c\right)$.

72 S. Sachdev, Quantum Phase Transitions (Cambridge Uni- 
versity, Cambridge, England, 1999).

73 E. Manousakis, Rev. Mod. Phys. 63, 1 (1991).

74 The function used in this interpolation is $T_{X}=$ $\sqrt{T_{\mathrm{N}}^{\mathrm{HF}}+T^{\prime 2}}$

75 V.L. Berezinskii, Zh. Eksp. Teor. Fiz. 59, 907 (1970).

76 J. Kosterlitz and D. Thouless, J. Phys. C 6, 1181 (1973).

77 For a further discussion, see S. Allen, H. Touchette, S. Moukouri, Y.M. Vilk, and A.M.-S. Tremblay, Phys. Rev. Lett. 83, 4128 (1999).

78 V.P. Gusynin, V.M. Loktev, and S.G. Shaparov, JETP 90, 993 (2000).

79 V.P. Gusynin, V.M. Loktev, R.M. Quick, and S.G. Sha- parov, Physica C 370, 239 (2002).

80 Note that in the strong coupling limit, one can integrate out the $\Phi$ field to obtain the effective action of the $\boldsymbol{\Omega}$ field without assuming AF short-range order [Eq. (14)]. To leading order in $t / U$, one recovers the action of the Heisenberg model ${ }^{57}$

81 R. Preuss, W. Hanke, and W. von der Linden, Phys. Rev. Lett. 75, 1344 (1995).

82 A. Moreo, S. Haas, A.W. Sandvik, and E. Dagotto, Phys. Rev. B 51, 12045 (1995).

83 H. Matsumoto and F. Mancini, Phys. Rev. B 55, 2095 (1997). 